\title{
6 Befreit Moral? Immanuel Kants autonomieorientierte Ethik
}

\author{
6.1 Der Kategorische Imperativ und seine Anwendung \\ 6.2 Einwände und Schwierigkeiten \\ 6.3 Versuch einer plausiblen Deutung der kantischen Ethik \\ 6.4 Philosophischer Kontext und Ausblick
}

Moralphilosophischer Empirismus: Die bisherige Darstellung neuzeitlicher Ethik war hinsichtlich der repräsentierten philosophischen Strömungen einseitig: Unter den neuzeitlichen Autoren wurden ausschließlich Vertreter des Empirismus in den Blick genommen (zur Unterscheidung zwischen Empirismus und Rationalismus siehe Kap.3.1). Sie teilen (will man sie nicht gar als Vernunftskeptiker interpretieren) ein instrumentelles Verständnis der praktischen Vernunft. Entsprechend bleiben ihnen im Hinblick auf die Interpretation der Moral nur zwei Optionen: Entweder sie halten an der traditionell angenommenen Verbindung zwischen Moral und Vernunft fest und interpretieren auch die Moral selbst instrumentell. Oder sie schwächen die Verbindung zwischen Moral und Vernunft $\mathrm{ab}$ und weisen der Moral (zusätzlich) eine andere Grundlage zu. Den ersten Weg gehen Vertragstheoretiker/innen wie Hobbes und die Utilitaristen. Den zweiten Weg gehen Moral-Sense-Theoretiker/innen, die moralische Unterscheidungen in Gefühlen begründet sehen. Hume steht sozusagen mit je einem Fuß in beiden Lagern, denn seine Moralphilosophie kombiniert natürliche Tugenden, die unmittelbarer Ausdruck emotionaler Einstellungen sind, mit künstlichen Tugenden, die primär einem institutionell umgelenkten rationalen Eigeninteresse entstammen.

Probleme des ethischen Instrumentalismus: An den bislang betrachteten Ansätzen lassen sich zudem generelle Schwierigkeiten des Empirismus ablesen. Instrumentalistische Theorien sind grundsätzlich nicht imstande, uns auf die letzten Ziele unseres Handelns zu verpflichten. Denn letzte Ziele können nicht wiederum instrumentell begründet werden. Soweit instrumentalistische Ethiken letzte Ziele nicht einfach als Gegenstand a-rationaler Willkürentscheidungen verstehen wollen (Dezisionismus), sollten sie diese Ziele zumindest als ohnehin unkontrovers oder jedenfalls als im Wesentlichen plausibel und unproblematisch ausweisen. Aber gibt es wirklich unkontroverse Vorschläge für letzte Ziele? Ist beispielsweise die Maximierung des subjektiven Wohlbefindens aller empfindungsfähigen Wesen ein unkontroverses Ziel? Und wenn es dies tatsächlich wäre - würde eine weitgehende faktische Übereinstimmung bezüglich dieses Ziels ausreichen, um auch moralische Verpflichtungen, Schuldzuschreibungen und gegebenenfalls sogar schmerzhafte Sanktionen zu rechtfertigen?

Probleme der Moral-Sense-Theorien: Ähnliche Fragen stellen sich auch im Hinblick auf die sentimentalistischen Theorien: Gibt es hinrei-

Begründung "letzter" Ziele? 
Von der Kontemplation zum Experiment chend große Ähnlichkeiten und hinreichend große Stabilität in unserem Gefühlsleben, um darauf eine Moraltheorie zu begründen? Entspricht eine auf unsere `natürlichen` Gefühlstendenzen begründete Moral demjenigen, was wir als gültige Moral anzuerkennen bereit sind? Angenommen, der Inhalt der Moral ließe sich tatsächlich aus unserem Gefühlsleben herleiten - könnten wir auf dieser Grundlage moralische Vorschriften, Verantwortung und Schuld zuschreiben? Wären wir dann nicht vielmehr zu der Annahme genötigt, dass diejenigen, die moralische Erwartungen verletzen, letztlich an einer emotionalen Störung leiden und müssten wir dementsprechend nicht psychotherapeutische Interventionen an die Stelle moralischer Kritik setzen? Sowohl in seiner instrumentalistischen wie in seiner sentimentalistischen Variante fällt es dem Empirismus offenbar schwer, die Normativität moralischer Erwartungen auf Begriffe zu bringen. Freilich könnte es sein, dass sich Normativität ohnehin schwer oder gar nicht philosophisch fassen lässt. Es gibt jedoch auch Gründe für die Vermutung, dass der Empirismus - jedenfalls in den bislang vorgestellten Ansätzen - von vornherein einen Zugang zum Phänomen der menschlichen Praxis wählt, der Normativität gar nicht erst in den Blick geraten lässt. Um diese Vermutung plausibel zu machen, sei etwas weiter zurückgegriffen.

Rückblick: Die primäre Erkenntnishaltung der antiken Naturphilosophie und Metaphysik war die der kontemplativen Betrachtung. Theoria bedeutet eigentlich Schau. Gegenstand der Kontemplation war die freie, ungebundene Natur, nicht die unter kontrollierten Bedingungen experimentell untersuchte Natur. Unterschiede zwischen der Naturerkenntnis und der Erkenntnis der menschlichen Praxis sieht Aristoteles vor allem im Hinblick auf die Variabilität des Gegenstandes. In beiden Bereichen meint er jedoch dieselben Strukturen zweckgerichteter Wirksamkeit zu entdecken. Der spätmittelalterliche Nominalismus unterminiert die Basis der teleologischen Wesensphilosophie. An die Stelle der theoretischen Schau, die in der kontemplativen Betrachtung freier Natur deren Wesen $\mathrm{zu}$ entschlüsseln sucht, tritt eine aktiv ins Naturgeschehen eingreifende experimentelle Naturforschung. Die neuzeitliche Naturwissenschaft untersucht, wie vor allem Francis Bacon hervorhebt, nicht die freie, sondern die zielgerichtet manipulierte Natur. An dieser objektivierten, unter kontrollierten Bedingungen untersuchten Natur lässt sich die Annahme der Wirksamkeit von Entelechien nicht bestätigen. Stattdessen lassen sich der Natur ihre kausalen Gesetzmäßigkeiten entreißen. Die Methode der experimentellen Naturwissenschaft basiert auf der Hypothese, dass sich alle Naturprozesse rein kausal aus vorausliegenden Ursachen erklären lassen.

Praxisvergessenheit des Empirismus: Die Wissenschaftspraxis des neuzeitlichen Empirismus hat insofern die passive, kontemplative Erkenntnisform der antiken Theoria-Tradition hinter sich gelassen. Allerdings wird die aktive Rolle, die der Experimentator im Erkenntnisprozess durch seine zielgerichteten Interventionen in Naturzusammenhänge spielt, in den erkenntnistheoretischen Überlegungen des neuzeitlichen Empirismus nur begrenzt reflektiert. Hume blendet die aktiven gegenstandskonstitutiven Leistungen des Erkenntnissubjekts weitgehend aus 
(abgesehen nur von der Wirksamkeit psychologischer Assoziationsmechanismen bei der Kombination von Eindrücken). Er entwickelt seine Erkenntnistheorie gleichsam aus der Perspektive eines Kinozuschauers, hinter dessen Rücken ohne sein Zutun der Film seiner Erfahrungen abgespult wird. Das führt paradoxerweise dazu, dass einerseits sogar Kausalität selbst fragwürdig wird, weil dasjenige, was einem passiven Beobachter erscheint, der die eigenen geplanten Interventionen in den Weltverlauf ausblendet, immer nur die zeitliche Abfolge von Phänomenen, aber niemals das Wirksamwerden irgendeiner kausalen Kraft ist. Andererseits will Hume gleichwohl auch noch den Bereich der menschlichen Praxis und des menschlichen Gefühlslebens nach dem Muster kausal erklärbarer Naturphänomene interpretieren. Er vergleicht, wie schon erwähnt, seine Herangehensweise mit der eines Anatomen, der die Strukturen unseres Gefühlslebens ausmisst, um dessen Funktionsweise zu erklären, und rechnet es sich als Verdienst an, auch die Moral zum Gegenstand der experimentellen Untersuchung und Beobachtung zu machen, nachdem sich diese Methode auf dem Gebiet der Naturforschung bereits hundert Jahre früher durchgesetzt habe (Hume 2007, 1.Intr.7, S. 4 f.). In der Perspektive von Empirist/innen wie Hume (und bereits Hobbes) wird mithin auch das menschliche Handeln zu einem Naturprozess, in dem unsere Verlangen die Rolle von >Triebfedern`spielen, die, nach Maßgabe mehr oder weniger korrekter Annahmen über die Außenwelt und vermittelt durch die Hebel, Seilzüge und Umlenkrollen einer rein instrumentellen Vernunft, unser Verhalten in Bewegung setzen.

Erklärungsperspektive und Deliberationsperspektive: In dieser auf quasi-naturwissenschaftliche Erklärung observierter Gefühle, Entscheidungen oder Handlungen gerichteten Perspektive kann praktische Freiheit nun aber ebenso wenig in den Blick geraten wie eigentlich - Humes eigener Einsicht zufolge - Kausalität. Es ist damit auch nicht die Perspektive, in der die Normativität praktischer Gründe sichtbar werden kann. Denn die Frage, ob ein vermeintlicher Handlungsgrund tatsächlich ein guter Grund für eine bestimmte Handlung ist, stellt sich uns ausschließlich in der Perspektive von Teilnehmer/innen eines deliberativen Prozesses, die den Horizont ihres Handelns als offen erfahren. Die deliberative Perspektive unterscheidet sich damit strukturell von der Perspektive erklärender Naturbeobachtung. Insbesondere interpretieren wir unsere eigenen Neigungen und Verlangen (ebenso wie die an uns gerichteten Verlangen anderer Personen) in der deliberativen Perspektive nicht als Naturkräfte, die durch uns wie durch ein Räderwerk hindurchwirken. Wir begreifen sie vielmehr als (implizite) Ansprüche auf Berücksichtigung, zwischen denen wir abwägen und denen wir uns gegebenenfalls auch verweigern können. Selbst dort, wo wir nachträglich die Stichhaltigkeit von Handlungsgründen bewerten, nehmen wir virtuell diese deliberative Perspektive ein. 
Tipp Kant lesen

Zitiert wird Kant meist nach der Akademieausgabe. Sie ist weiterhin im Druck und zudem in Gestalt des Bonner Kant-Korpus auch in elektronischer Form frei und mit praktischer Suchfunktion verfügbar (https:// korpora.zim.uni-duisburg-essen.de/kant/). Auf zentrale Werke Kants wird häufig durch Siglen verwiesen, so auch im Folgenden; siehe das Verzeichnis am Ende des Kapitels.

Raum für Freiheit und Moralität

Kants Abkehr vom kontemplativen Erkenntnisideal: Kants kritische Philosophie zielt darauf, Empirismus und Rationalismus miteinander zu vermitteln und dadurch auch hartnäckige philosophische Probleme als Probleme verständlich zu machen, die durch die Struktur und die Grenzen unseres Erkenntnisvermögens bedingt sind. Die spezifische Form der kantischen Vermittlung von Empirismus und Rationalismus rückt einen weiteren Schritt vom erkenntnistheoretischen Modell passiver Kontemplation ab. Kant betrachtet Erkenntnis als Tätigkeit, die die Welt, soweit sie für uns zur Erscheinung kommt, wesentlich prägt. In seiner Transzendentalphilosophie soll die Vernunfttätigkeit selbstreflexiv ihr eigenes Funktionieren aufklären und ihre eigenen Grenzen ausmessen. Dadurch soll auch Raum für die Möglichkeit praktischer Freiheit und Moralität geschaffen werden. Im Interesse eines möglichst einfachen Zugangs werden die tieferen philosophischen Grundlagen der kantischen Ethik allerdings zunächst zurückgestellt. Die Darstellung wird sich im ersten Schritt ganz auf eine der Formulierungen des von Kant vorgeschlagenen Moralprinzips beschränken. In einem zweiten Schritt werden einige miteinander verhakte Interpretationsprobleme und Komplikationen untersucht, die sich bei dem Versuch der praktischen Orientierung an diesem Moralprinzip ergeben. Erst im Anschluss daran wird knapp auf den größeren Kontext der kantischen Philosophie Bezug genommen.

\section{1 | Der Kategorische Imperativ und seine Anwendung}

Der gute Wille: Der Hauptteil von Kants 1785 erschienener Schrift Grundlegung zur Metaphysik der Sitten (GMS) beginnt mit folgender, häufig zitierter Passage:

GMS, S. 393 "Es ist überall nichts in der Welt, ja überhaupt auch außer derselben zu denken möglich, was ohne Einschränkung für gut könnte gehalten werden, als allein ein guter Wille. Verstand, Witz, Urtheilskraft und wie die Talente des Geistes sonst heißen mögen, oder Muth, Entschlossenheit, Beharrlichkeit im Vorsatze als Eigenschaften des Temperaments sind ohne Zweifel in mancher Absicht gut und wünschenswerth; aber sie können auch äußerst böse und schädlich werden, wenn der Wille, der von diesen Naturgaben Gebrauch machen soll und dessen eigenthümliche Beschaffenheit darum Charakter heißt, nicht gut ist. Mit den Glücksgaben ist es eben so be- 
wandt. Macht, Reichthum, Ehre, selbst Gesundheit und das ganze Wohlbefinden und Zufriedenheit mit seinem Zustande unter dem Namen der Glückseligkeit machen Muth und hiedurch öfters auch Übermuth, wo nicht ein guter Wille da ist, der den Einfluß derselben aufs Gemüth und hiemit auch das ganze Princip zu handeln berichtige und allgemein=zweckmäßig mache; ohne zu erwähnen, daß ein vernünftiger unparteiischer Zuschauer sogar am Anblicke eines ununterbrochenen Wohlergehens eines Wesens, das kein Zug eines reinen und guten Willens ziert, nimmermehr ein Wohlgefallen haben kann, und so der gute Wille die unerlaßliche Bedingung selbst der Würdigkeit glücklich zu sein auszumachen scheint."

Diese Passage lässt erkennen, worin Kant den Gegenstand der moralischen Beurteilung erblickt: Es ist der Wille einer Person, der letztlich Hume als moralisch gut oder böse zu beurteilen ist. Kant unterscheidet klar zwischen der äußeren Richtigkeit der Handlung, die er später auch »Legalität« nennt (KpV, S. 71 f.), und ihrer Moralität. Eine Handlung - oder genauer: der sie regierende Wille - ist nur dann moralisch gut, wenn nicht nur pflichtgemäß, sondern auch aus Pflicht bzw. aus Achtung vor dem (Moral-)Gesetz gehandelt wird. Hinsichtlich des Gegenstandes der moralischen Beurteilung stimmt Kant also in gewissem Umfang mit Hume überein. Auch in Kants Verständnis zielt die moralische Beurteilung wesentlich auf den subjektiven Willen bzw. den Charakter, für den die Handlungen einer Person lediglich Indizien liefern. Auch durch die Bezugnahme auf die Figur des unparteiischen Zuschauers knüpft Kant explizit an die Moral-sense-Tradition an. Zugleich macht die Passage jedoch eine wesentliche Differenz, nicht nur zum Eudaimonismus antiker Tugendethiken, sondern auch zur humeschen Tugendethik deutlich. Denn den natürlichen Tugenden Humes liegen tatsächlich »Eigenschaften des Temperaments « zugrunde; Eigenschaften also, die nach Kants Auffassung als bloße "Naturgaben" stets ambivalent bleiben und nur durch die Leitung des »reinen und guten Willens« veredelt werden können. Für die Moral kommt es nicht auf das natürliche Temperament, sondern allein auf den selbstbestimmten Willen einer Person an.

Wesen des Kategorischen Imperativs: Was aber dient als Kriterium des »reinen und guten Willens«? Ebenso wie der Utilitarismus postuliert Kant ein oberstes Moralprinzip, an dem sich der Wille orientieren soll. Kant nennt dieses Prinzip den Kategorischen Imperativ. Kant sucht zu zeigen, dass das Moralprinzip ein notwendiges Prinzip der praktischen Vernunft darstellt. Praktische Vernunft ist nach Kants Auffassung also nicht nur instrumentelle Vernunft; sie ist nicht auf die Beurteilung von Mitteln für gegebene Zwecke beschränkt und daher auch nicht (wie von Hume angenommen) ssterik. Sie enthält vielmehr (genauer: konstituiert sich selbst als praktische Vernunft durch) ein praktisches Prinzip, das (in Verbindung mit unseren Maximen, s. u.) selbst handlungsorientierende Kraft hat. Weil wir Menschen nicht immer schon von Natur aus vollkommen vernünftig handeln, nimmt dieses Prinzip für uns den Charakter einer normativen Forderung - eines Imperativs - an. Kategorisch nennt Kant einen Imperativ genau dann, wenn seine Verbindlichkeit unabhängig davon besteht, ob seine Befolgung den Wünschen oder Interessen des Ad- 
ressaten entgegenkommt. In diesem spezifischen Sinn sind Kategorische Imperative sunbedingt`. Hypothetische Imperative drücken hingegen nur aus, was Adressat/innen tun müssen, soweit sie bestimmte Ziele verfolgen.

\begin{tabular}{|c|c|c|}
\hline \multicolumn{2}{|c|}{ Imperative } \\
\hline hypothetische & kategorische \\
\hline technische & pragmatische & moralische \\
\hline Regeln der Geschicklichkeit & Ratschläge der Klugheit & Gebote der Sittlichkeit \\
\hline $\begin{array}{c}\text { Wie erreiche ich } \\
\text { (effizient), Ziel Z? }\end{array}$ & Wie lebe ich glücklich? & $\begin{array}{c}\text { Was muss ich unbedingt } \\
\text { tun und lassen? }\end{array}$ \\
\hline
\end{tabular}

Unbedingtheit der Moral

GMS, S. 402
Dabei unterscheidet Kant noch einmal zwischen zwei Arten hypothetischer Imperative: Technische Imperative geben Bedingungen für die Erreichung beliebiger Ziele an, die Menschen verfolgen mögen oder auch nicht. Pragmatische Imperative geben demgegenüber Orientierungen für ein gutes und glückliches Leben der Akteurin oder des Akteurs. Sie sind damit auf ein Ziel gerichtet, das nach Kants (wie nach Aristoteles') Auffassung alle Menschen tatsächlich verfolgen, das allerdings inhaltlich nicht klar umrissen ist. Kant nennt die technischen Imperative auch »Regeln der Geschicklichkeit«, die pragmatischen auch »Ratschläge der Klugheit« (GMS, S. 416). Darin drückt sich eine gewisse Unsicherheit aus, ob der Begriff "Imperativ« in Bezug auf die hypothetischen Imperative angemessen ist. Denn da hypothetische Imperative letztlich "gar nicht gebieten, d.i. Handlungen objektiv als praktisch-notwendig darstellen" handele es sich eher um "Anrathungen (consislia) als Gebote (praecepta) der Vernunft" (GMS, S.418). Die unbedingte (kategorische) Verbindlichkeit ist für Kant ein wesentliches Merkmal moralischer Forderungen. Darin liegt eine klare Absage an den Instrumentalismus, der letztlich immer nur »Regeln der Geschicklichkeit« begründen kann, wie an den Eudaimonismus, der sich mit »Ratschlägen der Klugheit« begnügt. Folgt man Kant, so berührt beides den Bereich der Moral noch gar nicht. Denn das Wesen der Moral ist nicht durch bestimmte Inhalte, sondern eben durch ihren unbedingten Verbindlichkeitsanspruch definiert.

Die Gesetzesformel des Kategorischen Imperativs: In der Grundlegung schlägt Kant zwar unterschiedliche Formulierungen des Kategorischen Imperativs vor, die in der Sekundärliteratur zu verschiedenen `Formeln zusammengefasst werden. Er vertritt jedoch die Auffassung, dass alle Formulierungen im Hinblick auf die durch das Prinzip erhobenen Forderungen äquivalent sind. Eine der Formulierungen der sogenannten Gesetzesformel des Kategorischen Imperativs lautet:

\section{"[I]ch soll niemals anders verfahren als so, daß ich auch wollen könne, meine Ma- xime solle ein allgemeines Gesetz werden."}

Maximen und Gesetze: Wie können wir uns die Orientierung an diesem Prinzip vorstellen? Anders als etwa das Moralprinzip des Handlungsutilitarismus schreibt der Kategorische Imperativ nicht unmittelbar ein be- 
stimmtes Handlungsziel vor, an dem das Handeln sich direkt orientieren soll. Vielmehr ist die Orientierung am Kategorischen Imperativ auf eine noch näher zu klärende Weise über Zwischenprinzipien vermittelt, die Kant Maximen nennt. Die Maximen sind es wiederum, die dem Handeln eine bestimmte Ausrichtung geben. In dieser Mehrstufigkeit der moralischen Orientierung scheint einerseits eine gewisse Parallele zum Regelutilitarismus zu liegen. Dass Kant die moralische Beurteilung nicht unmittelbar auf Handlungen, sondern auf die Maximen bezieht, entspricht aber anderseits auch seiner Auffassung, dass der Wille der eigentliche Gegenstand der moralischen Beurteilung ist. Die Maximen sind nämlich nicht einfach nur Handlungsregeln, denen das Handeln einer Person (möglicherweise rein zufällig) entsprechen könnte. Vielmehr sind sie dasjenige, was den Willen einer Person wesentlich bestimmt (Höffe 1977, S. 367). Es sind allgemeine Grundsätze, an denen sich Personen in ihrem Handeln orientieren, die für diese Personen also generelle Handlungsgründe darstellen. In der Grundlegung finden sich mehrere Beispiele für solche Grundsätze. Eine der Maximen, die Kant als Beispiel anführt, lautet etwa: "Wenn ich mich in Geldnoth zu sein glaube, so will ich Geld borgen und versprechen, es zu bezahlen, ob ich gleich weiß, es werde niemals geschehen " (GMS, S.422). Ebenso könne es "sich jemand zur Maxime machen, keine Beleidigung ungerächt zu erdulden« (KpV, S. 19) oder sich »alle Mittel des Wohllebens in der Absicht auf den Genuß anzuschaffen und zu erhalten« (MdS, S. 432). Kant definiert die Maximen als subjektive Handlungsprinzipien. Mit der Kennzeichnung der Maximen als subjektiv will Kant ausdrücken, dass noch nicht ausgemacht ist, ob die Maximen auch als allgemeingültige und in diesem Sinne objektive Handlungsgrundsätze gerechtfertigt werden können. Objektive Handlungsprinzipien nennt Kant Gesetze.

Das Moralprinzip als 'Maximenfilter: Auf welche Weise gelangen wir nun von subjektiven Maximen zu objektiven praktischen Gesetzen? Anders als das Prinzip »Maximiere das Glück aller Lebewesen!« legt uns der Kategorische Imperativ nicht direkt auf Inhalte unseres Handelns fest. Kant betont, dass es sich beim Kategorischen Imperativ um ein rein formales Moralprinzip handelt. Die Funktionsweise dieses Prinzips lässt sich mit dem Bild eines Maximenfilters erläutern. Maximen, die den Filter passieren, sind moralisch akzeptabel; wir dürfen sie verfolgen. Maximen, die den Filter nicht passieren, sind unmoralisch; wir dürfen uns nicht an ihnen orientieren. Durch den Test von Maximen, die wir uns >vormoralisch zu eigen gemacht haben oder zu eigen machen könnten, gelangen wir also einerseits zu moralischen Erlaubnissen, andererseits zu moralischen Verboten. Falls es zu dem Verbotenen nur eine einzige Alternative gibt, können wir daraus indirekt auch Gebote gewinnen (da es verboten ist, Verträge zu brechen, ist es geboten, Verträge einzuhalten).

Zweistufiger Maximentest: Um eine meiner (möglichen) Maximen am Kategorischen Imperativ zu testen, muss ich mich fragen, ob ich wollen kann, dass sich alle Personen in allen vergleichbaren Situationen an derjenigen Maxime orientieren, auf deren Grundlage ich zu handeln erwäge. Wenn dies der Fall ist, darf ich mir diese Maxime zu eigen machen (bzw. 
Mehrstufiger Maximentest

GMS, S. 422
GMS, S. 424 "Einige Handlungen sind so beschaffen, daß ihre Maxime ohne Widerspruch nicht einmal als allgemeines Naturgesetz gedacht werden kann; weit gefehlt, daß man noch wollen könne, es sollte ein solches werden. Bei anderen ist zwar jene Unmöglichkeit nicht anzutreffen, aber es ist doch unmöglich zu wollen, daß ihre Maxime zur Allgemeinheit eines Naturgesetzes erhoben werde, weil ein solcher Wille sich selbst widersprechen würde."

darf sie beibehalten), anderenfalls nicht. Dabei lassen sich, bleibt man im Bild des Maximenfilters, gewissermaßen zwei Filterstufen unterscheiden:

Die Beantwortung der Frage, ob wir eine bestimmte Maxime als allgemeines Gesetz wollen können, muss demnach in zwei Schritten erfolgen. Wir prüfen zunächst das Denken-Können und dann das Wollen-Können eines der zu prüfenden Maxime entsprechenden allgemeinen Gesetzes.

Vier Beispiele: Kant sucht in der Grundlegung den Maximentest an vier Beispielen zu demonstrieren. Zwei Beispielmaximen scheitern ihm zufolge im ersten, zwei im zweiten Prüfungsschritt. Dabei bezieht sich jeweils eine Maxime auf das Verhalten gegenüber anderen Personen und eine auf das Verhalten gegenüber der eigenen Person. Kants Beispiel für eine auf das Verhalten gegenüber anderen bezogene Maxime, die als allgemeines Gesetz nicht einmal denkbar ist, ist die schon zitierte Maxime des falschen Versprechens (»wenn ich mich in Geldnoth zu sein glaube, so will ich Geld borgen und versprechen, es zu bezahlen, ob ich gleich weiß, es werde niemals geschehen «). Verträge schließen zu können ist ja überhaupt nur möglich, so lange potentielle Vertragspartner/innen davon ausgehen, dass das Prinzip der Vertragstreue gilt, wonach Verträge einzuhalten sind. Die Strategie eines geplanten Vertragsbruchs ist nur als Strategie eines Trittbrettfahrers denkbar, der von der allgemeinen Erwartung der Vertragstreue profitiert, indem er die Vertragspartner über seine wahren Intentionen täuscht. Als allgemeine Praxis würde die Maxime jedoch die Erwartung der Vertragstreue und damit eine notwendige Bedingung ihrer eigenen Möglichkeit aufheben:

"[D]ie Allgemeinheit eines Gesetzes, daß jeder, nachdem er in Noth zu sein glaubt, versprechen könne, was ihm einfällt, mit dem Vorsatz, es nicht zu halten, würde das Versprechen und den Zweck, den man damit haben mag, selbst unmöglich machen, indem niemand glauben würde, daß ihm was versprochen sei, sondern über alle solche Äußerung als eitles Vorgeben lachen würde."

Von anderen Maximen können wir uns hingegen widerspruchsfrei vorstellen, dass sie allgemein als Handlungsprinzipien akzeptiert wären. Trotzdem würden wir, Kant zufolge, von einigen dieser Maximen nicht wollen, dass sie als allgemeine Gesetze Geltung hätten. Ein Beispiel ist die Maxime der Gleichgültigkeit gegenüber der Not anderer: der Grundsatz, anderen Personen, die »mit großen Mühseligkeiten zu kämpfen haben « in ihrer Not nicht beizustehen, obwohl das im Rahmen des Möglichen läge. Zwar lässt sich denken, dass eine solche Maxime allgemein befolgt würde. Wir können aber nach Kants Auffassung nicht ernsthaft wollen, dass alle sich an einer Maxime der Gleichgültigkeit gegenüber der 
Not anderer orientieren, da »der Fälle sich doch manche ereignen können«, wo wir selbst der "Liebe und Teilnehmung" anderer Personen bedürfen. Daher würden wir uns vernünftigerweise nicht »alle Hoffnung des Beistandes« in solchen Fällen »rauben« lassen wollen (GMS, S. 423). Als Beispiele für subjektive Prinzipien, die primär das Verhalten gegenüber der eigenen Person betreffen, nennt Kant zum einen die Maxime, "wenn das Leben bei seiner längeren Frist mehr Übel droht, als es Annehmlichkeit verspricht, es mir abzukürzen" (GMS, S. 422), also sich das Leben zu nehmen; zweitens die Maxime »lieber dem Vergnügen nachzuhängen, als sich mit Erweiterung und Verbesserung seiner glücklichen Naturanlagen zu bemühen« (GMS, S. 423). Die Maxime der hedonistisch motivierten Selbsttötung wäre nach Kants Auffassung nicht einmal als allgemeines Gesetz zu denken, sie soll also schon im ersten Prüfungsschritt scheitern. Die Maxime, die Bequemlichkeit vor Selbstentfaltung setzt, sei dagegen zwar zu denken, aber nicht als allgemeines Gesetz zu wollen. Kants Argumentation ist in diesem Zusammenhang knapp und nicht sehr klar; sie soll an dieser Stelle nicht weiter diskutiert werden.

Pflichttypen: Dass Kant vier Beispielmaximen anführt, die im vom Kategorischen Imperativ gebotenen Test allesamt scheitern, ist kein Zufall. Nur im Falle des Scheiterns eines Maximentests werden nämlich moralische Pflichten oder »Gesetze« sichtbar, die den Charakter von Verboten oder Geboten haben. Den vier Beispielen entsprechen dabei vier Typen von Pflichten:

\begin{tabular}{|l|l|l|}
\hline \multicolumn{2}{|l|}{ Pflichttypen und Beispiele } \\
\hline & Vollkommene Pflichten ... & Unvollkommene Pflichten ... \\
\hline $\begin{array}{l}\text {... gegenüber } \\
\text { sich selbst }\end{array}$ & $\begin{array}{l}\text { Verbot hedonistisch motivierten } \\
\text { Suizids }\end{array}$ & $\begin{array}{l}\text { Verbot hedonistisch motivierter } \\
\text { Selbstverwahrlosung }\end{array}$ \\
\hline $\begin{array}{l}\text {... gegenüber } \\
\text { anderen }\end{array}$ & $\begin{array}{l}\text { Verbot lügenhaften Verspre- } \\
\text { chens / Gebot der Vertragstreue }\end{array}$ & $\begin{array}{l}\text { Verbot der Indifferenz gegenüber } \\
\text { der Not anderer / Hilfsgebot }\end{array}$ \\
\hline
\end{tabular}

Aus dem Scheitern selbstbezogener Maximen ergeben sich Pflichten gegenüber sich selbst, aus dem Scheitern von Maximen, die das Verhalten gegenüber anderen regieren, ergeben sich sozialethische Pflichten gegenüber anderen. In beiden Pflichtkategorien unterscheidet Kant wiederum zwischen vollkommenen Pflichten und unvollkommenen Pflichten. Kant zufolge stellen diejenigen moralischen Pflichten vollkommene Pflichten dar, die sich in der ersten Phase des Tests ergeben - Verbote und Gebote also, die daraus resultieren, dass bestimmte Maximen als Grundlagen einer allgemeinen Handlungsorientierung noch nicht einmal 'gedacht « werden können. Diejenigen moralischen Pflichten, die sich in der zweiten Phase des Tests ergeben, weil wir bestimmte Maximen zwar als sallgemeine Gesetze` denken, aber nicht wollen können, sind hingegen unvollkommene Pflichten (siehe Abb. 6.1).

Ethischer Absolutismus: Kants Unterscheidung zwischen vollkommenen und unvollkommenen Pflichten ist folgenreich. Vollkommene Pflichten sind unter allen Umständen zu befolgen. Unvollkommene Pflichten dürfen hingegen zurückgestellt werden, wenn sie mit anderen, 
Abb. 6.1: Gesetzesformel des Kategorischen Imperativs als sMaximenfilters

\section{Von Maximen...}

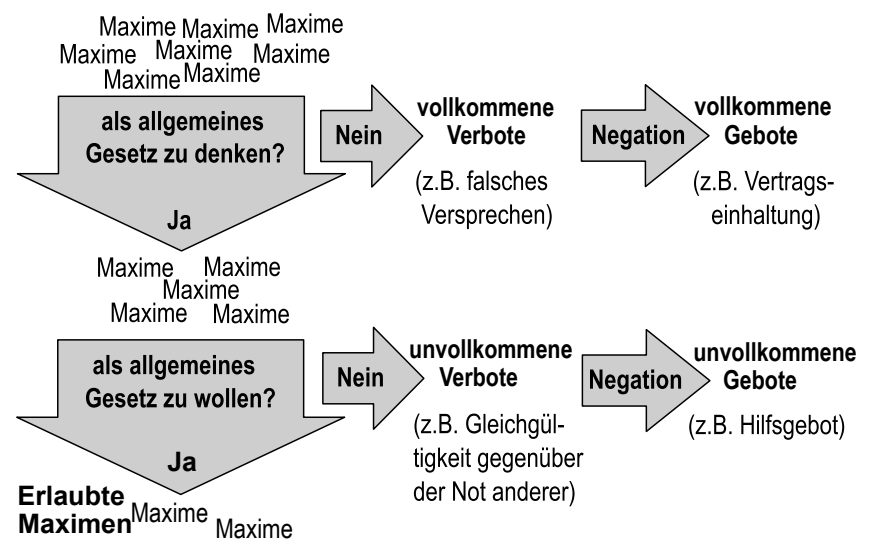

...zu Gesetzen

vorrangigen moralischen Pflichten kollidieren, also nicht gleichzeitig mit ihnen befolgt werden können. Wie Kant in der Metaphysik der Sitten klarstellt, dürfen freilich auch die unvollkommenen Pflichten nicht etwa um vormoralischer Neigungen willen zurückgestellt werden (MdS, S. 390). Eine dieser Aussage widersprechende Äußerung in einer Anmerkung der Grundlegung ist sehr wahrscheinlich nur ein später revidierter Lapsus Kants (Kersting 1993, S. 189 ff.; anderer Auffassung ist Höffe 2000, S. 214), zumal sie von Kant in der betreffenden Anmerkung selbst ausdrücklich als vorläufig gekennzeichnet wird (GMS, S.421). Die Auffassung, dass moralische Pflichten keinerlei Ausnahmen dulden, wird auch als ethischer Absolutismus bezeichnet; in Bezug auf die vollkommenen Pflichten ist Kant also ein Absolutist.

\section{Absolutismus, Deontologie und Kategorizität}

Von manchen Autor/innen wird auch der Begriff "Deontologie« so definiert, dass er auf einen Absolutismus hinausläuft (klassisch Broad 2013, S. 206 f.). Dies entspricht nicht der von Frankena und Rawls eingeführten Standarddefinition des Deontologiebegriffs, die auch im vorliegenden Band verwandt wird. Geht man von dieser Definition aus, so muss eine deontologische Ethik nicht notwendigerweise absolutistisch sein. Ferner ist die vollständige Ausnahmslosigkeit der Verbindlichkeit von Pflichten auch keine Implikation des kategorischen Charakters moralischer Pflichten im Sinne von Kant. Kategorisch gelten Kant zufolge neben den vollkommenen auch die unvollkommenen Pflichten: Die Verbindlichkeit beider Arten von Pflichten hängt nämlich nicht davon ab, ob ihre Befolgung im vormoralischen Interesse der handelnden Person liegt. Deshalb dürfen beide nicht um vormoralischer Neigungen willen zurückgestellt 
werden. Das schließt aber nicht aus, dass sie aus moralischen Gründen zurückgestellt werden dürfen - Letzteres ist die Möglichkeit, die Kant für die unvollkommenen Pflichten einräumt, aber für die vollkommenen bestreitet. Dass Kant in Bezug auf die vollkommenen Pflichten einen ethischen Absolutismus vertritt, folgt also weder aus dem Umstand, dass seine Ethik deontologisch ist, noch aus dem Umstand, dass er den moralischen Pflichten kategorische Verbindlichkeit zuschreibt.

Konsequenzen des Absolutismus: Kants Unterscheidung zwischen vollkommenen und unvollkommenen Pflichten mag auch erklären, weshalb Kant später in dem kurzen Aufsatz Über ein vermeintes Recht aus Menschenliebe zu lügen (AA, Bd. 8, S. 423-430) zu dem Schluss kommt, man dürfe eine Person mit Mordabsichten nicht über den Aufenthaltsort von deren prospektivem Opfer belügen. Kants Beurteilung dieses auf Benjamin Constant zurückgehenden Beispiels ist viel diskutiert worden. Sie erscheint jedoch konsequent, wenn man annimmt, dass die Wahrhaftigkeitspflicht eine vollkommene, ausnahmslos verbindliche Pflicht darstellt, während es sich bei der Hilfsverpflichtung nur um eine unvollkommene Liebespflicht handelt.

Moralischer Wert: In scharfem Gegensatz zu Hume betont Kant, dass dem Handeln nur dann ein moralischer Wert zukommt, wenn es nicht nur pflichtgemäß, sondern aus Pflicht vollzogen wird: Die ausschlaggebende Motivation muss eben in der Pflichterfüllung selbst liegen, nicht in moralunabhängigen Neigungen der handelnden Person. Die einzige Form moralisch guter Handlungen ist nach Kants Auffassung gerade diejenige, die in Humes Moralphilosophie bestenfalls als eine mit den Grundlagen seiner Theorie kaum vereinbare Ausnahmeerscheinung möglich scheint (Hume ist unentschlossen hinsichtlich der Frage, ob ein Handeln aus reinem Pflichtgefühl möglich ist; vgl. Hume 2007, 3.2.1.7, S. 308). Während Hume ein Handeln aus natürlicher Menschenfreundlichkeit, natürlicher Großzügigkeit, natürlicher Furchtlosigkeit umstandslos als tugendhaft bewertet, mag ein solches Handeln nach Kants Auffassung zwar häufig pflichtgemäß sein, hat aber keinerlei moralischen Wert, eben insofern es bloßen Naturanlagen entstammt und nicht der vernünftigen Orientierung am moralischen Gesetz. Dass nicht nur zufälligerweise pflichtgemäß bzw. äußerlich richtig gehandelt wird, sondern tatsächlich aus Pflicht und damit moralisch gut, ist gerade dort am wahrscheinlichsten, wo Personen ihren natürlichen Neigungen zuwiderhandeln.

\section{2 | Einwände und Schwierigkeiten}

Bis zu diesem Punkt ging es vor allem darum, ein Vorverständnis davon zu gewinnen, wie der Kategorische Imperativ als Moralprinzip dem Willen überhaupt eine Richtung geben könnte. Bei näherem Hinsehen wird eine Reihe von Unklarheiten und Schwierigkeiten sichtbar. Im Folgenden werden zunächst vier gängige Einwände gegen das von Kant vorgeschla- 
Vier verbreitete Einwände

Schiller 1943 ff., Bd. 20, S. 357 gene Moralprinzip skizziert. Im Anschluss soll verdeutlicht werden, dass im Hinblick auf die Frage, wie wir uns genau am Kategorischen Imperativ orientieren sollen, verschiedene Interpretationen möglich sind. Auf dieser Grundlage wird dann eine Lesart vorgeschlagen, die von den zuvor aufgeführten Einwänden möglichst wenig betroffen ist; allerdings um den Preis, etwas komplizierter zu sein als konkurrierende Lesarten.

1. Egozentrismus: Kants Kategorischer Imperativ lässt sich als kritische Weiterentwicklung der Goldenen Regel verstehen. Als Moralprinzip erscheint die Goldene Regel problematisch, weil sie eine bestimmte Form von egozentrischer Beurteilung nicht überwinden kann. Kant betont zwar zu Recht, dass sein Moralprinzip nicht mit der Goldenen Regel identisch ist. Trotzdem lässt sich fragen, ob Kant das Egozentrismusproblem der Goldenen Regel vollständig ausräumen kann.

2. Formalismus: Was man von einem Moralprinzip mindestens erwarten kann, ist, dass es überhaupt irgendeine Orientierung bietet. Einige Autoren, darunter Hegel, bezweifeln jedoch, dass der Kategorische Imperativ dem Handeln überhaupt eine Richtung weisen kann. Bestimmte Lesarten der kantischen Ethik können diesen Zweifel tatsächlich nähren.

3. Legalistischer Rigorismus: Viele Interpret/innen halten die von Kant unterstellte Absolutheit derjenigen moralischen Gesetze, die aus dem ersten Schritt der Maximenprüfung resultieren, für unplausibel - für in problematischer Weise regelfetischistisch oder rigoristisch. Ihrer Auffassung nach sprechen unsere moralischen Intuitionen dafür, dass auch die von Kant für >vollkommen gehaltenen Pflichten offen für Abwägungen sein sollten (dass wir z. B. einen prospektiven Mörder belügen dürfen, wenn dies die einzige Möglichkeit darstellt, den von ihm Verfolgten zu retten).

4. Moralischer Rigorismus: Rigorismus wird Kant auch noch in einer anderen Bedeutung vorgeworfen: Im Sinne einer Kritik an seiner Auffassung, dass ein Handeln aus natürlichen Neigungen grundsätzlich ohne moralischen Wert sei, auch dann, wenn es sich um prosoziale Neigungen wie Wohlwollen, Menschenliebe oder Großzügigkeit handelt. Der stark von Kant beeinflusste Schiller hat dem Einwand ironischen Ausdruck gegeben:

\section{Gewissensskrupel}

"Gern dien' ich den Freunden, doch thu ich es leider mit Neigung

Und so wurmt mir oft, daß ich nicht tugendhaft bin."

\section{Decisum}

"Da ist kein anderer Rath, du mußt suchen sie zu verachten, Und mit Abscheu alsdann thun, wie die Pflicht dir gebeut."

Im Interesse der Unterscheidbarkeit wird diese Form des Rigorismus im Anschluss an Höffe als moralischer Rigorismus bezeichnet, die zuvor besprochene Form als legalistischer Rigorismus (Höffe 1990, S. 190 ff.; Höffe 2000, S. 217 f.). 


\subsection{1 | Egozentrismus-Problem und Goldene Regel}

Die Goldene Regel: Die sogenannte Goldene Regel ist im Zusammenhang mit Hobbes bereits kurz erwähnt worden (siehe Kap.3.2). Sie stellt ein sehr früh und in verschiedenen Varianten in zahlreichen religiösen und juristischen Überlieferungen nachweisbares Moralprinzip dar (Neusner/ Chilton 2009; Wattles 1996). Allgemeines Kennzeichen des Prinzips ist, dass es die Legitimität des eigenen Verhaltens von einer bestimmten Art des (gedankenexperimentellen) Rollentauschs abhängig macht. So soll eine sich zum Handeln anschickende Person prüfen, ob sie die von ihr geplante Handlung auch dann gutheißen würde, wenn sie in der Rolle einer der von der Handlung betroffenen Personen wäre (’negative` Fassung der Goldenen Regel):

"Was du nicht willst, dass man dir tu, das füg' auch keinem andern zu!"

Oder die Person ist aufgefordert, aktiv dasjenige zu tun, was sie selbst von der Person, die sie jetzt ist, erwarten würde, wenn sie in der Situation einer der Handlungsbetroffenen wäre (’positive` Fassung der Goldenen Regel):

"Was Du willst, dass andere Dir tun, tue innen!"

Modelle der Unparteilichkeit: Die Goldene Regel verdankt ihre (begrenzte) Plausibilität weit verbreiteten moralischen Intuitionen der Unparteilichkeit. Es erscheint unfair, an das eigene Handeln (einschließlich des Unterlassens von aktiven Handlungen) andere Maßstäbe anzulegen als an das Handeln anderer Personen. Eben dies - das Messen mit zweierlei Maßstab - ist es, was die Goldene Regel in jeder ihrer Fassungen verbieten möchte (vgl. Singer 2001; Wimmer 1980, S. 254 ff., 290 ff.). Die Goldene Regel stellt allerdings nur einen Versuch unter mehreren dar, die Intuition der Unparteilichkeit auf den Begriff zu bringen. Der im Kontext des humeschen Sentimentalismus erwähnte unparteiische Beobachter stellt einen weiteren Versuch dar; ebenso der Kategorische Imperativ Kants, der von John Rawls vorgeschlagene Urzustand (siehe Kap. 7.2.2) oder die diskursethische Orientierung am Grundsatz der allgemeinen Konsensfähigkeit (z. B. Habermas’ »Universalisierungsprinzip«; 1996, S. 60).

Kants Einwände gegen die Goldene Regel: In der gelebten Moral und den Strukturen moralischen Urteilens (vgl. Kohlberg 1981, Bd.1) spielt die Goldene Regel zweifellos eine zentrale Rolle. Warum also geben sich so viele Moralphilosoph/innen - darunter Kant - nicht damit zufrieden, sondern suchen nach alternativen Modellen der Unparteilichkeit? Die Goldene Regel drückt die Intuition der Unparteilichkeit offenbar zumindest dann nicht angemessen aus, wenn man sie als die Aufforderung versteht, unmittelbar die Perspektive der konkreten Handlungsbetroffenen einzunehmen. Entsprechend interpretiert Kant die Goldene Regel, wo er im Sinne eines argumentum ad absurdum ausführt, dass ein Verbrecher diese Regel gegen einen Richter anführen könnte, der ihn zu einer Strafe verurteilen wolle (GMS, S.430, Anm.). Das Argument des Verbrechers wäre dann offenbar, dass auch der Richter, wenn er in der Haut des Ver- 
brechers steckte, freigesprochen werden wollte, und ihn daher entsprechend dem Prinzip »Was Du nicht willst...« nicht verurteilen dürfe. Kant wendet gegen die Goldene Regel zweitens ein, dass sich auf ihrer Basis keine Pflichten gegenüber der eigenen Person rechtfertigen ließen. Drittens ließen sich auch keine »Liebespflichten gegen andere« begründen, denn, so Kant, »mancher würde es gerne eingehen, daß andere ihm nicht wohlthun sollen, wenn er es nur überhoben sein dürfte, ihnen Wohlthat zu erzeigen« (ebd.).

Intendierte Revision der Goldenen Regel: Kants Kategorischer Imperativ soll diese Probleme anscheinend dadurch vermeiden, dass er nicht die Akzeptabilität einer konkreten Einzelhandlung aus der Perspektive des konkreten Betroffenen zum Kriterium der moralischen Richtigkeit der Handlung macht, sondern die Akzeptabilität der zum allgemeinen Gesetz erhobenen Handlungsmaxime aus der Perspektive einer handelnden Person, die sich selbst als Gesetzgeber universeller Moralgesetze versteht. Der Kategorische Imperativ müsste sich demnach von der Goldenen Regel unterscheiden, indem er zweierlei klarstellt:

1. Es geht bei der moralischen Beurteilung niemals (nur) um die Akzeptabilität einer isoliert betrachteten Einzelhandlung, sondern (stets auch) um die einer allgemeinen Handlungsweise (Universalität des Gegenstands).

2. Es ist nicht die Perspektive einer einzelnen konkreten Person entscheidend, sondern eine unparteiische Perspektive, die die Perspektive der Akteurin bzw. des Akteurs selbst ebenso umfasst wie die aller möglichen Betroffenen (Universalität des Standpunkts).

Ebenen der Universalisierung
Beide Punkte sind bedeutsam. Der erste drückt im Grunde eine Bedingung ethischer Argumentation überhaupt aus. Rationales Argumentieren über die moralische Richtigkeit einer konkreten Handlung in einer konkreten Situation ist nur auf der Basis von Gründen möglich, die grundsätzlich auf alle Handlungen in allen Situationen zutreffen, die im Hinblick auf ihre allgemeinen (in universellen Begriffen ausdrückbaren) Merkmale identisch sind. Würde man nämlich annehmen, moralische Qualitäten hafteten an der numerischen Identität ihrer Gegenstände (so dass, was in Situation S1 richtig ist, in Situation S2 falsch sein könnte, obwohl es keinerlei qualitative Unterschiede zwischen $S 1$ und S2 gibt), käme die ethische Argumentation zum Erliegen. Im Rahmen der kantischen Ethik ist die Universalität des Beurteilungsgegenstandes notwendig, um den ersten Schritt der vom Kategorischen Imperativ gebotenen Maximenprüfung zu ermöglichen. Denn eine `Denkunmöglichkeit ‘ wie im Fall des falschen Versprechens kann sich überhaupt nur in Bezug auf allgemeine Gesetze einstellen. Die Universalität des Standpunkts ist wiederum wichtig um zu verhindern, dass die partikulare Perspektive einer einzelnen konkreten Person - einer Person mit spezifisch-persönlichen, eventuell sogar bizarren, Präferenzen - zum maßgeblichen moralischen Standpunkt verabsolutiert wird. Dabei wäre nicht nur die Verabsolutierung der konkreten Betroffenenperspektive problematisch, sondern ebenso die Verabsolutierung der Perspektive einer konkreten handelnden Person, die sich zwar per Gedankenexperiment in die objektive Lage ei- 
nes/einer Handlungsbetroffenen versetzt, diese Lage aber ausschließlich auf der Grundlage ihrer eigenen persönlichen Präferenzen und Wertvorstellungen beurteilt.

Alternative Lesarten der Goldenen Regel: Die erste Problematik wird in Kants Gerichtsbeispiel thematisiert. Kant interpretiert hier die Goldene Regel so, dass sie auf eine Verabsolutierung der Betroffenenperspektive hinausläuft: Es sind die konkreten, subjektiv-persönlichen Präferenzen des Verbrechers, die darüber entscheiden, wie der Richter ihn behandeln soll. Nicht weniger problematisch wäre freilich eine Verabsolutierung der Akteursperspektive also in diesem Fall der empirischen Präferenzen des Richters. Zwar verbietet die Goldene Regel eine Entscheidung gemäß dem unmittelbaren Eigeninteresse des Handelnden: Der Richter darf den Verbrecher beispielsweise nicht einfach deshalb verurteilen, weil dies für seine Karriere nützlich wäre. Eine direkte egoistische Handlungsorientierung wird durch die Orientierung an der Goldenen Regel unterbunden. Denkbar ist freilich auch eine subtilere Form des Akteurs-Egozentrismus: Nehmen wir beispielsweise an, der Richter sei eine unsichere, von zahlreichen Ängsten geplagte Person. Versetzt er sich in die Lage des Verbrechers - Mitglied einer kriminellen Motorradgang, die sich aktuell in einer gewaltsamen Fehde mit einer konkurrierenden Gruppe befindet - so wäre sein innigster Wunsch der nach einer vieljährigen Gefängnisstrafe, die ihm ermöglicht, in der relativen Sicherheit des Gefängnisses den Ausgang der Fehde auszusitzen, bevor er wieder in Freiheit entlassen wird. Orientiert er sich nun in dem Sinne an der Goldenen Regel, dass er von demjenigen ausgeht, was er selbst wollen würde, wenn er sich äußerlich in der Lage des Delinquenten befände, so würde er ihm eine lange Gefängnisstrafe auferlegen. Gerichtsbeispiele sind freilich problematisch, weil hier ja primär positive Rechtsnormen zu beachten sind. Ein gängiges Alternativbeispiel ist das eines Sadomasochisten, der sich fragt, ob er einer anderen Person Schmerz zufügen darf. Versetzt er sich in dessen $o b$ jektive Situation, geht dabei aber weiter von seinen unmittelbaren persönlichen Präferenzen aus, könnte er die Schmerzzufügung mit der Goldenen Regel für vereinbar halten. Diese problematische Verabsolutierung der Akteursperspektive ließe sich zwar vermeiden, wenn man die von der Goldenen Regel geforderte Rollenübernahme so interpretiert, dass die handelnde Person sich nicht nur in die äußere Situation der Handlungsbetroffenen versetzen, sondern auch deren subjektive Präferenzen und Wertvorstellungen übernehmen soll, um auf Basis von deren Präferenzen und Werthaltungen die eigenen Handlungsoptionen zu beurteilen. Damit fiele man jedoch wieder auf die ebenso problematische Option zurück, die Perspektive der oder des Handlungsbetroffenen (z. B. von Kants Verbrecher) zu verabsolutieren. Plausibel kann also nur eine Interpretation sein, die weder die Perspektive der einen noch der anderen Person unkritisch verabsolutiert. Einer solchen Interpretation näher kommt etwa Nelsons sogenanntes Abwägungsgesetz »Handle nie so, daß du nicht auch in deine Handlungsweise einwilligen könntest, wenn die Interessen der von ihr Betroffenen auch deine eigenen wären" (Nelson $1970 \mathrm{ff}$., Bd. 4, S. 133, Hervorh. MW). Zu klären bleibt dann freilich, auf welche Weise in denjenigen Fällen, in denen sich die Perspektiven von Handeln- 
den und (möglicherweise auch mehreren) Betroffenen unterscheiden, ein Konsens aller Beteiligten hergestellt werden kann, so dass Akteur/in und Betroffene die Handlungsweise gemeinsam akzeptieren können.

Egozentrismusproblem und Kategorischer Imperativ: Die Goldene Regel drückt die Intuition der Unparteilichkeit also nicht in völlig befriedigender Weise aus. Kann der Kategorische Imperativ die genannten Schwächen restlos überwinden? Darüber lässt sich jedenfalls streiten. Kants Einwand gegen die Goldene Regel, dass sie keine "Liebespflichten gegen andere « rechtfertigen könne, weil "mancher [...] es gerne eingehen " würde, »daß andere ihm nicht wohlthun sollen, wenn er es nur überhoben sein dürfte, ihnen Wohlthat zu erzeigen« (GMS, S. 430, Anm.), scheint grundsätzlich auch auf den Kategorischen Imperativ zuzutreffen (Parfit 2011, Bd. 1, S. 321 ff.). Denn Kants Begründung für die Annahme, dass wir die Maxime der Gleichgültigkeit gegenüber der Not anderer nicht als allgemeines Gesetz wollen können, soll ja eben auf der Annahme basieren, dass wir uns durch ein solches Gesetz "selbst alle Hoffnung des Beistandes [...] rauben« (GMS, S. 423) würden. Für Personen, die tatsächlich bereit sind, ohne diese Hoffnung zu leben - das heißt für genau die Personen, auf die sich Kant in seiner Kritik der Goldenen Regel bezieht -, könnte demnach auch der Kategorische Imperativ keine »Liebespflichten« begründen. Die von Kant als Kriterium angeführte Überlegung, ob »ich wohl damit zufrieden sein [könnte], daß meine Maxime [...] als ein allgemeines Gesetz [...] gelten solle« (GMS, S. 403) kann also die subtilere Form des Akteurs-Egozentrismus jedenfalls nicht ohne Weiteres vermeiden. Die Gefahr liegt in der Verabsolutierung der persönlichen Präferenzen der handelnden Person in Situationen, in denen sich diese Präferenzen von denen der Handlungsbetroffenen unterscheiden.

\subsection{2 | Zwei Varianten des Formalismus-Einwands}

Lassen sich zu allen Handlungen verallgemeinerbare Maximen finden?
Hegels Formalismuseinwand: Hegel hat gegen Kants Kategorischen Imperativ eingewandt, dass er eine reine Leerformel sei. Nun betont zwar Kant selbst, dass es sich dabei um ein rein formales Moralprinzip handelt. Konkrete Handlungsinhalte sollen erst durch die Maximen ins Spiel kommen und werden durch den Kategorischen Imperativ sozusagen nur ausgefiltert. Hegel behauptet jedoch, dass der Moralfilter in Wahrheit gar nicht selektiv sei: Jede Maxime lasse sich nämlich so formulieren, dass sie der Anforderung des Kategorischen Imperativs genüge. Es gebe "gar nichts, was nicht auf diese Weise zu einem sittlichen Gesetz gemacht werden könnte« (Hegel 1970, Bd. 2, S. 461). Dieser Einwand weist auf ein ernstes Problem hin: Handlungsregeln lassen sich unterschiedlich formulieren, und ein und dieselbe konkrete Handlung könnte grundsätzlich Ausdruck unterschiedlicher Maximen sein (z. B.: »Ich will lügen«, »Ich will lügen, wenn ich dadurch mein Ziel erreiche«, »Ich will lügen, wenn dies die einzige Möglichkeit ist, das Leben eines Verfolgten zu retten«, etc.). Wenn wir Maximen nun so verstehen würden, dass es sich dabei auch um extrem spezifisch formulierte Regeln handeln dürfte (so lange sie nur in universellen Termini formuliert sind, also etwa keine Eigen- 
namen oder konkrete Zeit- oder Ortsangaben enthalten), ließe sich (sofern wir auch noch das "wollen können« in bestimmter Weise interpretieren; s. u.) tatsächlich jede intendierte Handlung als Ausdruck einer Maxime verstehen, die eine handelnde Person als ‘allgemeines Gesetz` »wollen kann« - ganz einfach deswegen, weil sie aufgrund der sehr spezifischen Formulierung gar nicht zu erwarten braucht, dass sich jemals eine zweite Anwendungssituation ergibt. Auch eine >Denkunmöglichkeit von Maximen könnte sich bei sehr spezifisch formulierten Maximen kaum einstellen. Um Hegels Version des Formalismuseinwands gegen die Gesetzesformel des Kategorischen Imperativs zurückweisen, scheint ein anspruchsvollerer Maximenbegriff nötig zu sein.

Formalismuseinwand als Zirkularitätseinwand: Ein Formalismusproblem würde sich allerdings nicht nur bei allzu spezifischen, sondern auch bei extrem allgemeinen Maximenformulierungen ergeben. Versteht man als »Maxime« nämlich, wie der vom lateinischen »maximus" ("größter«) abgeleitete Begriff nahelegt, als das allgemeinste, >oberster Handlungsprinzip einer Akteurin, so stellt sich die Frage, ob für eine moralisch vorbildliche Person nicht der Kategorische Imperativ selbst die Maxime sein muss. Tatsächlich lassen sich Ausführungen in Kants 1793 erschienenen Schrift Die Religion innerhalb der Grenzen der bloßen Vernunft so verstehen, dass wir in jeder Entscheidung nur die Wahl zwischen zwei Maximen haben: der Maxime der Selbstliebe und dem in Maximenform gebrachten Kategorischen Imperativ (AA, Bd. 6, S. 36; vgl. Tugendhat 1993, S. 124 f.). Diese Deutung wird auch durch Formulierungen des Kategorischen Imperativs nahegelegt, die sich bereits in der Grundlegung finden, etwa:

\section{"[H]andle nur nach derjenigen Maxime, durch die du zugleich wollen kannst, daß sie ein allgemeines Gesetz werde."}

Diese Aufforderung würde offenbar zu einem Begründungszirkel und damit tatsächlich zu einem leeren Formalismus führen. Denn der Kategorische Imperativ könnte uns nur immer wieder erneut auferlegen, gemäß dem Kategorischen Imperativ zu handeln, ohne dass wir je herausfinden könnten, welche konkreten Handlungsinhalte damit gegeben sind.

\subsection{3 | Legalistischer Rigorismus}

Wie der `legalistische Rigorismus`, der in der erwähnten Schrift Über ein vermeintes Recht... zum Ausdruck kommt, zu bewerten ist, hängt von Annahmen darüber $a b$, welche fallbezogenen Urteile sich aus einer normativ-ethischen Theorie ergeben sollten. Diese Bewertung ist ethisch nicht neutral. In allen Ethiken, die die moralische Richtigkeit einer Handlung nicht (nur) von den Folgen abhängig machen, sondern (auch) von der Übereinstimmung des Handlungstyps mit generellen Normen oder Prinzipien, besteht grundsätzlich die Möglichkeit, dass eine konkrete Handlung, die durch eine Einzelnorm geboten wird, problematisch oder gar anstößig erscheint (entweder intuitiv oder vor dem Hintergrund allgemeinerer ethischer Prinzipien). Dies trifft etwa auch auf den Regeluti- 
Beurteilung des Absolutismus litarismus zu (Smart 1973). Es stellt sich dann stets die Frage, ob und unter welchen Bedingungen Ausnahmen von der Regel zugelassen werden sollen. Die von Kant im Hinblick auf die vollkommenen Pflichten vertretene absolutistische Auffassung verhindert eine Verantwortungsdiffusion, wie sie etwa bei einer rein konsequenzorientierten Beurteilung drohen würde: Im Rahmen der kantischen Ethik ergibt sich eindeutig, dass allein der Mörder Verantwortung für die Ermordung seines Opfers trägt, nicht derjenige, der den Mord durch eine wahre Aussage ermöglicht hat. (Hätte Letzterer gelogen und dadurch - ungewollt - dem Mörder doch in seinem Plan geholfen, trüge er hingegen eine Mitverantwortung.) Andererseits widersprechen einige ihrer Konsequenzen deutlich gegen verbreitete moralische Intuitionen. Darüber hinaus lässt sich fragen, warum die ausnahmslose Verbindlichkeit eines bestimmten Moralgesetzes ausgerechnet von der Frage abhängen soll, ob die Verallgemeinerung einer gesetzeswidrigen Maxime unmöglich zu denken oder nur unmöglich zu wollen ist. Für die moralische Bedeutung dieser Unterscheidung wären jedenfalls zusätzliche Gründe anzuführen. Zu fragen ist jedoch auch, ob die von Kant selbst gezogenen rigoristischen Konsequenzen wirklich unvermeidlich sind oder ob sie nicht wiederum durch eine plausiblere Interpretation des Maximenbegriffs vermieden werden können (s. u.).

\subsection{4 | Moralischer Rigorismus}

Bei der zweiten Form von Rigorismus, die Schiller beklagt, handelt es sich um ein weniger tiefgreifendes Problem. Denn man kann durchaus mit Kant der Auffassung sein, dass ein Handeln aus `natürlichen` Neigungen nicht moralisch verdienstvoll ist und dass keine Neigung unser Handeln ungeprüft bestimmen sollte, weil auch ein durch prosoziale Neigungen motiviertes Handeln im konkreten Fall falsch sein kann - und kann gleichwohl die Kultivierung prosozialer Neigungen für moralisch begrüßenswert und einen Einklang zwischen Neigung und moralischer Pflicht für erstrebenswert halten (Tugendhat 1993, S. 116 ff.). Die wesentlichen Festlegungen der kantischen Moralphilosophie schließen diese Position jedenfalls nicht aus.

\section{3 | Versuch einer plausiblen Deutung der kantischen Ethik}

Lesarten des Kategorischen Imperativs: Im vorigen Abschnitt wurden Einwände gegen Kants Moralprinzip skizziert. Dabei ist deutlich geworden, dass die Gültigkeit der Einwände oft von bestimmten Interpretationen dieses Moralprinzips und seiner Anwendung abhängt. Betrachten wir noch einmal die bereits angeführte Formulierung:

GMS, S. 402,

Unterstreichungen MW
"[I]ch soll niemals anders verfahren als so, daß ich auch wollen könne, meine

Maxime solle ein allgemeines Gesetz werden." 
Hier sind unter anderem drei Fragen zu klären, die allerdings miteinander verhakt sind:

1. Wer genau ist das »Ich«, dessen »Als-allgemeines-Gesetz-wollen-Können« der Maxime ausschlaggebend ist? Ist jede Akteurin und jeder Akteur als konkretes Individuum gemeint? Steht das »Ich« stellvertretend für eine idealisierte, z. B. besonders rationale Person? Oder steht es repräsentativ für eine reale oder wiederum irgendwie idealisierte moralische Gemeinschaft?

2. Wie ist das "Wollen« dieses »Ich" genau zu verstehen? Handelt es sich schlicht um die faktischen Präferenzen realer Personen? Oder muss das maßgebliche »Wollen« bereits irgendwelchen Rationalitätsbedingungen genügen?

3. Wie sind »Maximen« genau beschaffen? Handelt es sich um extrem generelle oder um extrem spezifische Handlungsregeln oder um Regeln einer mittleren Allgemeinheit? Oder sind Maximen gar nicht als einfache Handlungsregeln zu verstehen, sondern als Prinzipien mit einer komplexeren Struktur?

Kant hat in seinen Schriften zwar Hinweise, aber keine ganz eindeutigen und befriedigenden Antworten auf diese Fragen gegeben. Unabhängig von Kants eigenen Auffassungen lässt sich fragen, welche (Kombinationen von) Antworten am meisten Sinn ergeben (vgl. zum Folgenden ausführlicher Werner 2003, S. 134-150).

"lch" und "Wollen-Können": Was die erste und die zweite Frage betrifft, gilt jedenfalls Folgendes: Einerseits kann das maßgebliche Wollen kein vollständig vernünftiges Wollen eines schlechthin rationalen Wesens sein. Denn als schlechthin vernünftiges Wollen bestimmt Kant gerade dasjenige, das am Kategorischen Imperativ orientiert ist, so dass die Antwort zu einem Zirkelproblem führen würde (gemäß der zweiten Variante des Formalismusvorwurfs): Wir müssten das Ergebnis der Maximenprüfung schon in Händen haben, um mit der Prüfung beginnen zu können. Setzt man andererseits das maßgebliche »Ich« umstandslos mit dem konkreten Individuum eines Akteurs und das maßgebliche Wollen mit seinen empirischen Präferenzen gleich, droht wiederum das Problem des subtilen Akteurs-Egozentrismus, das oben im Zusammenhang mit Kants Kritik an der Goldenen Regel verdeutlicht wurde: Soweit die empirischen Präferenzen verschiedener Akteure sich unterscheiden, wäre es problematisch, wenn auf der Basis der Präferenzen eines Akteurs allgemeine Gesetze begründet würden, die für alle gleichermaßen gelten sollen. Soweit der zweite Schritt der Maximenprüfung betroffen ist, kämen dann als Ergebnis der Maximenprüfung in gewissem Sinne gar keine wirklich rallgemeinen - soll heißen: aus der Perspektive aller Handelnden gleichermaßen verbindlichen - Gesetze mehr zustande.

"lch" als virtuelles "Wir": Aus eben diesem Grund müsste freilich auch jede Person einsehen können, dass sie die sozusagen höherstufige Maxime (M $\left.{ }^{\text {privat}}\right)$, nach Maximen zu handeln, die zwar sie selbst aufgrund ihrer persönlichen Präferenzen als allgemeine Gesetze wollen kann, die andere Personen auf Basis ihrer persönlichen Präferenzen jedoch nicht als allgemeine Gesetze wollen können, selbst nicht als all- 
"Ich" als Platzhalter der Moralgemeinschaft gemeines Gesetz wollen kann. Denn die allgemeine Befolgung von $M^{\text {privat }}$ würde zu einer Auflösung des Begriffs eines allgemeinen Moralgesetzes als eines aus der Perspektive aller Adressaten gleichermaßen verbindlichen Prinzips führen. Aus der Perspektive verschiedener Akteure könnten vielmehr jeweils unterschiedliche und unvereinbare Moralgesetze begründet erscheinen. Eine Maxime wie $M^{\text {privat }}$ als allgemeines Gesetz zu wollen würde bedeuten zu wollen, dass andere Personen nach `Gesetzen handeln, welche die eigenen Präferenzen unberücksichtigt lassen. Dies erscheint jedoch widersprüchlich: Ich kann nicht zugleich wollen, dass sallgemeiner Gesetze - das heißt solche, die für alle Moralsubjekte verbindlich sind - allein von meinen eigenen Präferenzen und allein von den Präferenzen anderer abhängen. Die einzig akzeptable Alternative scheint darin zu liegen, beim zweiten Schritt der Maximenprüfung nicht Privatinteressen zugrunde zu legen, sondern sich im Bewusstsein der unterschiedlichen Präferenzen und Wertorientierungen um eine unparteiische Beurteilung der Auswirkungen einer Verallgemeinerung der zu prüfenden Maxime zu bemühen. Dass die maßgebliche Beurteilungsperspektive tatsächlich nicht die eines einzelnen konkreten Individuums, sondern die unparteiliche Perspektive der moralischen Gemeinschaft insgesamt ist, wird auch durch die sogenannte Reich der Zwecke-Formel des Kategorischen Imperativs nahegelegt, der zufolge »[d]as vernünftige Wesen [...] sich jederzeit als gesetzgebend in einem durch Freiheit des Willens möglichen Reich der Zwecke betrachten« muss. Kant erläutert allerdings nicht, auf welche Weise wir uns genau aus unvollkommenen Individuen mit unterschiedlichen Bedürfnissen, Interessen und Wertanschauungen in die "Glieder« derjenigen idealisierten moralischen Gemeinschaft verwandeln können, die Kant "Reich der Zwecke" nennt. Klar ist jedoch, dass das Ziel in der Einigung auf Kriterien liegt, die von allen Gliedern gleichermaßen akzeptiert werden können. Unsere Aufgabe wäre offenbar, uns angesichts unterschiedlicher empirischer Präferenzen auf eine gemeinsame Perspektive zu verständigen.

Problem der Maximenspezifikation: Das vielleicht am häufigsten diskutierte Problem im Hinblick auf die Orientierung am kategorischen Imperativ besteht in dem Umstand, dass Handlungen unterschiedlich beschrieben werden können und daher die Kopplung zwischen Maximen und Handlungen weniger starr ist, als Kant anzunehmen scheint. William David Ross formuliert das Problem als grundlegenden Einwand gegen Kants Moralprinzip:

»Kant's error seems to lie in this: Any individual act is an instance of a class of acts which is a species of a wider class which is a species of a still wider class; we can set no limit to the degrees of specification which may intervene between the summum genus sact and the individual act. For example, if $C$ tells a lie to the would-be-murderer, this falls (i) under the sub-species slies told to murderous persons`, (ii) under the species `lies`, (iii) under the genus `statements. Kant pitches, arbitrarily, on the middle one of these three classes, and since acts of this class are generally wrong, and are indeed always prima facie wrong, he says that the particular lie is wrong. But the man who tells the lie may well retort to Kant »Why should the test of universalizability be applied to my act regarded in this very abstract way, simply as a lie?« [...] The test of universalizability applied at 
one level of abstractness condemns the act; applied at another level of abstractness it justifies it. And since the principle itself does not indicate at what level of abstractness it is to be applied, it does not furnish us with a criterion of the correctness of maxims, and of the rightness of acts that conform to them."

(Ross 1954, S. 32 f.)

Ob dieser Einwand gegen den Kategorischen Imperativ und vergleichbare Verallgemeinerungsprinzipien sozusagen stödlich ‘ ist, wird kontrovers diskutiert (vgl. z. B. Potter/Timmons 1986; Wimmer 1980; im Kontext des Regelutilitarismus Singer 1963). Der Interpretation des Maximenbegriffs kommt in diesem Zusammenhang zentrale Bedeutung zu. Dass eine Klärung dieses Begriffs nötig ist, hat bereits die Diskussion der Vorwürfe des Formalismus und des legalistischen Rigorismus deutlich gemacht.

Maximen als subjektive (Prima-facie-)Gründe: Wesentliches Element einer möglichst plausiblen Deutung des Maximenbegriffs ist die Feststellung, dass es sich bei Maximen nicht einfach um mögliche Handlungsbeschreibungen oder beliebige Handlungsregeln handelt, unter die die Handlung mehr oder weniger zufällig fällt. Maximen sind vielmehr subjektive Gründe für bestimmte (Klassen von) Handlungen: Sie drücken aus, was diese (Klassen von) Handlungen aus Sicht der Akteurin oder des Akteurs tatsächlich geboten oder potentiell wertvoll erscheinen lässt (z. B., dass es sich um die Einhaltung eines Versprechens handelt, oder dass die Handlung notwendig ist, um einen unschuldig Verfolgten $\mathrm{zu}$ retten). Weil Maximen nicht beliebig wählbar sind, sondern reale (vorläufige) Handlungsgründe ausdrücken, können sich Akteur/innen nicht einfach irgendwelche verallgemeinerungsfähigen Formulierungen ihrer Maximen aussuchen - mithin auch nicht diejenigen, deren Gesetzesform sie am ehesten würden wollen können. ( $\mathrm{Zu}$ prüfende) Maximen bringen vielmehr (vorläufige) Selbstbindungen der Akteurin bzw. des Akteurs zum Ausdruck - dasjenige, worum es ihr oder ihm bei einer Handlung wirklich geht. Wer lügt oder zu lügen geneigt ist, weil es ihm einfach Spaß macht, andere Personen hinters Licht zu führen, kann demnach nicht einfach behaupten, seine Maxime sei gewesen, $\left(\mathrm{M}^{1}\right)$ »einen unschuldig Verfolgten zu retten« oder $\left(\mathrm{M}^{2}\right)$ »zu lügen, wenn dies zur Rettung eines Unschuldigen unvermeidlich ist «, denn sowohl $\mathrm{M}^{1}$ als auch die einschränkende Bedingung in $\mathrm{M}^{2}$ sind kein authentischer Ausdruck der realen Gründe für eigene Handlungen oder Handlungstendenzen.

Prima-facie-Charakter: Zweitens scheint es plausibel - vermutlich gegen Kant - anzunehmen, dass alle explizit formulierten Maximen aufgrund ihres mehr oder weniger stark generalisierenden Charakters nur als Prima-facie-Gründe für konkrete Handlungen verstanden werden können. Da sie mit anderen Prima-facie-Gründen kollidieren können, sind sie nur unter Vorbehalt auf neue Situationen zu übertragen. Absolute Verbindlichkeit für alle Situationen hat dieser Deutung zufolge nur das formale Moralprinzip selbst. Entsprechend sollten demnach auch die aus der Maximenprüfung resultierenden spezifischeren Moralgesetze grundsätzlich nur als Prima-facie-Pflichten verstanden werden. Plausibler als Kants Annahme der Absolutheit eines Teils der moralischen Pflichten wäre es, im Fall einer Pflichtenkollision einen erneuten Verallgemeine- 
Maximengeflecht mit Vorrangregeln rungstest möglicher alternativer Priorisierungen kollidierender Pflichten durchzuführen.

Komplexe Maximen: Um einen solchen Test zu ermöglichen, ist es freilich drittens nötig anzunehmen, dass neben einfachen Maximen wie »Ich will nicht lügen« oder "Ich will Menschen in Not helfen" auch komplexe Maximen möglich sind, die dadurch zustande kommen, dass einfachere Maximen durch Vorrangregeln verbunden werden. Wir hätten dann, wie Klaus Steigleder ausführt,

"von einem Geflecht oder Netz von Maximen auszugehen, das sich aus den Entscheidungen eines Individuums ergibt und das Über- und Unterordnungen von ,Willensbestimmungen` verschiedenen Allgemeinheitsgrades [...] enthält. Das Spektrum reicht [...] von einer grundlegenden und obersten Maxime für die Wahl aller Zwecke überhaupt bis hin zu den Entscheidungen, konkretere Handlungen auszuführen, um bestimmte Ziele zu erreichen." (Steigleder 2002, S. 124)

Ausgehend von einfachen Maximen, die ihrerseits verallgemeinerbar sein müssen, in einem konkreten Fall aber nicht gleichzeitig verfolgt werden können, fragen sich Akteur/innen, welche komplexere Maxime sie als allgemeines Gesetz akzeptieren könnten. Höffe schlägt in ähnlicher Weise eine alternative Lösung für das in Kants Schrift Über ein vermeintes Recht... beschriebene Beispiel vor:

»Die Maxime wäre formal gesehen komplexer als die uns vertrauten einfachen Maximen. Nennen wir die einfache Maxime (die der Ehrlichkeit oder die der Hilfsbereitschaft, der Gleichgültigkeit usw.) eine Basismaxime, so bestünde die (die Pflichtenkollision lösende) Maxime erstens aus mehr als einer Basismaxime, und jede der Basismaximen müßte schon den Test des Kategorischen Imperativs bestanden haben; in die komplexe Maxime gehen nicht die Unehrlichkeit und die Gleichgültigkeit, sondern die Ehrlichkeit und das Hilfsgebot ein. Zweitens enthielte die komplexe Maxime eine Prioritätsregel für die Basismaximen. Im Verhältnis zu Ehrlichkeit und Hilfsbereitschaft würde die Regel formuliert, unter den Bedingungen A (etwa der Rettung menschlichen Lebens) sei die Verletzung der Ehrlichkeit zulässig. Wie auch immer am Ende diese komplexere Maxime lauten würde: ihre Minimalbedingung, um moralisch zu sein, läge in der Verallgemeinerungsfähigkeit der Prioritätsregel. Kurz: der Prioritätenkonflikt, schlage ich im Rahmen der kantischen Ethik vor, wird durch ein zweistufiges Verallgemeinerungsverfahren gelöst." (Höffe 1990, S. 195; vgl. Höffe 2000, S. 218 f.)

\section{4 | Philosophischer Kontext und Ausblick}

Explikation der Alltagsmoral: Die bisherige Darstellung war ganz auf (eine der Formeln von) Kants Moralprinzip und dessen >Anwendung beschränkt. Die Perspektive soll nun noch ein wenig erweitert werden. Kant selbst geht in der Grundlegung von »der gemeinen sittlichen Vernunfterkenntnis« (GMS, erster Abschnitt) aus, d.h. von unserem vorphilosophischen Moralverständnis. Diesem Alltagsverständnis lasse sich entnehmen, dass der gute Wille das einzig schlechthin Gute darstelle (s. o.) und dass der gute Wille durch die Orientierung an der moralischen Pflicht gekennzeichnet sei (GMS, S. 397). Moralische Pflicht wiederum sei durch 
ihre strikte Verbindlichkeit und die Allgemeinheit der Gesetzesform bestimmt: "Pflicht ist die Nothwendigkeit einer Handlung aus Achtung fürs Gesetz." (GMS, S. 400) »Nothwendigkeit« könne aber nur einem Moralprinzip zukommen, das keinerlei empirische Bestimmungen enthalte, denn sonst wäre es bedingt durch kontingente Umstände. Da es sich auch beim menschlichen Glück um einen empirischen, von kontingenten Situationsumständen und den Wechselfällen des Schicksals bestimmten Zustand handelt, wendet sich Kant vehement gegen alle Ethiken, die das moralische Sollen auf das Ziel der Glückseligkeit beziehen (GMS, S. 441 ff.; KpV, S. 92 f.). Er betont, dass Moral kein Instrument der Glückseligkeit sein kann; schon gar nicht der Glückseligkeit der handelnden Person. Wohl aber kann sie uns des Glücks würdig machen. Auch ist es vernünftig, zu hoffen, dass in einer jenseitigen Welt die Harmonie von Moral und Glückseligkeit wiederhergestellt werden könnte (KpV, S. 107 ff.). Wenn nun jedoch, wie sich dem Verständnis moralischer Pflicht entnehmen lässt, "nichts als die allgemeine Gesetzmäßigkeit der Handlungen überhaupt [...] allein dem Willen zum Prinzip dienen soll«, so ergibt sich daraus, dass ich "niemals anders verfahren [soll] als so, daß ich auch wollen könne, meine Maxime solle ein allgemeines Gesetz werden" (GMS, S. 402; vgl. S. 420 f.).

Synthetisch-praktische Sätze a priori: Kant gewinnt den Kategorischen Imperativ in der oben angeführten Formulierung also zunächst aus einer Explikation unseres vorphilosophischen Moralverständnisses. Zugleich betont er, dass es sich bei diesem Prinzip selbst nicht um ein analytischpraktisches, sondern um ein synthetisch-praktisches Prinzip a priori handelt (GMS, S. 420). Was ist damit gemeint? Kant unterteilt Aussagen generell in analytische und synthetische Aussagen sowie in Aussagen a priori und a posteriori (KrV, B, S. 27 ff.). Analytische Aussagen buchstabieren aus, was in Begriffen bereits enthalten ist (z. B.: »Ein Junggeselle ist ein unverheirateter Mann«). Synthetische Aussagen dagegen erweitern unser Wissen. »Der Erdkern enthält Eisen« ist eine synthetische Aussage, weil nicht schon aus der Bedeutung des Begriffs »Erdkern« folgt, dass er Eisen enthält. Um festzustellen, ob der Erdkern Eisen enthält, ist Erfahrung nötig. Deshalb ist diese synthetische Aussage zugleich eine Aussage a posteriori, während die Behauptung, dass ein Junggeselle unverheiratet ist, schon vor jeder empirischen Untersuchung bestätigt werden kann, allein auf der Grundlage des Verständnisses der verwandten Begriffe. Es handelt sich daher um eine analytische Aussage a priori. Empirist/innen wie Hume halten nur analytische Aussagen a priori und synthetische Aussagen a posteriori für möglich. Kant hingegen behauptet, dass es auch synthetische Aussagen oder Prinzipien a priori gibt: Solche, die zugleich erkenntniserweiternd sind und vor aller Erfahrung als gültig erkannt werden können. Hypothetische Imperative sind im Grunde analytische Sätze. Denn sie buchstabieren lediglich aus, was bereits in dem jeweiligen Wollen enthalten ist, sind also nicht erkenntniserweiternd (GMS, S. 417 ff.). Insofern ich ein Ziel tatsächlich will, will ich auch die dafür notwendigen Mittel ergreifen. Die Wahrheit dieser Annahme folgt aus dem Begriff des Willens, der (im Gegensatz zum bloßen Wünschen) den Entschluss impliziert, das Gewollte unter den gegebenen 
Moral als Bedingung praktischer Selbstbestimmung
Umständen zu realisieren. Der Kategorische Imperativ ist ein synthetischer Satz a priori. Denn er buchstabiert nicht einfach aus, was bereits (vor der Maximenprüfung) in meinem Willen enthalten war, sondern schreibt dem Willen gegebenenfalls eine neuartige Richtung vor. Synthetisch a priori ist er, weil er die Kraft dazu nicht aus irgendwelchen empirischen Erkenntnissen schöpft, sondern allein aus der Idee eines freien Vernunftwesens (s. u.).

Transzendentalphilosophischer Hintergrund: Dass es synthetische Sätze a priori gibt, ist eine zentrale Annahme der kantischen Transzendentalphilosophie. Wie aber sind solche Aussagen möglich? Anders als frühere Idealisten und Rationalisten unterstellt Kant nicht die Existenz eingeborener Ideen, die uns vor aller sinnlichen Erfahrung mit inhaltlichen Erkenntnissen versorgen würden. Auch unterstellt er nicht, sondern bestreitet, dass wir durch synthetisch-apriorische Urteile oder Prinzipien Zugang zu einer schlechthin `objektiven`, das heißt von unserer Erfahrung oder Praxis unabhängigen, Wirklichkeit erhielten. Kant will vielmehr zeigen, dass bestimmte synthetische Urteile und Prinzipien deshalb für uns als erkenntnis- und handlungsfähige Vernunftwesen gültig sind, weil sie für unsere Erkenntnis oder für unsere praktische Freiheit konstitutiv sind: Unsere Erkenntnis- oder Deliberationspraxis funktioniert nur auf ihrer Grundlage. Weil es sich um Bedingungen der Möglichkeit unserer Erkenntnis bzw. unserer praktischen Freiheit handelt, sind sie für uns a priori gültig. Kants Konzeption apriorischer Bedingungen der Möglichkeit von theoretischer Erfahrung und praktischer Selbstbestimmung soll zwischen dem Empirismus und dem Rationalismus vermitteln. Eine zentrale Rolle spielt bei dieser Vermittlungsbemühung die Unterscheidung zwischen Inhalt und Form, und zwar in der theoretischen wie in der praktischen Philosophie. In der Kritik der reinen Vernunft sucht Kant zu zeigen, dass die Erkenntnis- und Verständnisleistungen von Sinnlichkeit, Verstand und Vernunft nur durch reine Formen, Kategorien und Ideen möglich sind. Auf diese Weise möchte Kant unter anderem das von Hume aufgeworfene Problem nach der Berechtigung von Kausalgesetzen lösen: Da Kausalität als solche kein Gegenstand der sinnlichen Erfahrung ist (was wir sinnlich wahrnehmen können, ist immer nur die zeitliche Abfolge von Phänomenen) werden Kausalbehauptungen für Hume problematisch; er sieht sie letztlich in psychologischen Mechanismen begründet. Kant hingegen sucht zu zeigen, dass die Kategorie der Kausalität eine Bedingung der Möglichkeit von Erfahrung darstellt, also eine apriorische Grundlage hat. Erfahrung ist jedoch nur durch ein Zusammenspiel der apriorischen Erkenntnisformen mit den durch unsere Sinne gelieferten Erkenntnisinhalten möglich: "Gedanken ohne Inhalt sind leer, Anschauungen ohne Begriffe sind blind" (KrV, A, S. 48; B, S. 75). Auf dem Gebiet der praktischen Philosophie verhält es sich ganz analog: Der synthetisch-apriorische Kategorische Imperativ gibt nur die Form unseres Willens vor, die empirischen Maximen sorgen gewissermaßen für die Inhalte unserer Willensbestimmung.

Moral als Autonomie: Wie erwähnt leitet Kant den Kategorischen Imperativ zunächst aus unserem moralischen Alltagsverständnis her. Dieses Alltagsverständnis könnte jedoch verzerrt oder gar gänzlich illusorisch 
sein. Kant begnügt sich denn auch nicht mit dieser Herleitung, sondern bemüht sich um eine transzendentalphilosophische Begründung des von ihm vorgeschlagenen Moralprinzips. Die Interpretation der betreffenden Passagen in der Grundlegung und der Kritik der praktischen Vernunft sind Gegenstand von Deutungskontroversen, die Kants Intentionen, seine verschiedenen Argumentationswege und deren jeweiligen Erfolg betreffen. Unstrittig ist jedoch, dass Kants Überlegungen um das Verhältnis zwischen Moral und praktischer Freiheit kreisen: Der freie Wille ist Kant zufolge als eine Art der Kausalität zu verstehen und muss daher (wie Kausalität überhaupt) nach allgemeinen Gesetzen wirken. Nach welchen Gesetzen aber könnte ein freier Wille wirken? Nur nach Gesetzen, die er sich selbst vorschreibt. Der freie Wille ist selbstgesetzgebend - autonom. Er orientiert sich also am Kategorischen Imperativ, denn dieser bindet den Willen an nichts anderes als an diejenigen allgemeinen Gesetze, denen ich selbst zustimmen könnte. Also sind Autonomieprinzip und Moralprinzip identisch. Kant überträgt damit einen Gedanken Rousseaus (1782, Bd.1, S. 187-360) aus der politischen Philosophie auf das Gebiet der Moralphilosophie. Wie Hobbes vertritt Rousseau eine Vertragstheorie politischer Autorität. Während jedoch Hobbes' Überlegungen ganz auf die Gewährleistung von Sicherheit für die einzelnen Bürger zielen, will Rousseau die Frage beantworten, wie wir in einer politischen Rechtsordnung zusammenleben können, ohne unsere Freiheit einzubüßen. Möglich ist dies, wenn wir den Gesetzen, denen wir uns unterordnen, als Mitglieder der Gemeinschaft selbst zustimmen können, weil sie Ausdruck des Allgemeinwillens, der volonté générale sind.

Möglichkeit der Willensfreiheit: Kant zufolge können wir uns nicht als freie Vernunftwesen verstehen, ohne uns am Moralprinzip zu orientieren und umgekehrt. (In der Sekundärliteratur wird diese Annahme häufig als reciprocity thesis bezeichnet; vgl. Allison 1986). Willensfreiheit und Moralität bedingen sich wechselseitig (GMS, S. 446 ff.; KpV, S. 3 ff.). Damit können wir zwar nicht das eine zugestehen und das andere bestreiten; aber warum sollten wir nicht beides bestreiten? Ist es vielleicht möglich, die Existenz der Willensfreiheit zu beweisen, um damit indirekt auch die Verbindlichkeit des Moralprinzips zu begründen?

\section{Willensfreiheit und Handlungsfreiheit}

Von menschlicher Freiheit wird in verschiedener Bedeutung gesprochen. Handlungsfreiheit lässt sich als die Abwesenheit äußerer Hindernisse bei der Realisierung der eigenen Handlungspläne verstehen. Sie kann etwa durch physischen Zwang oder natürliche Hindernisse eingeschränkt werden. Es ist diejenige Form von Freiheit, von der im Rahmen der hobbesschen Ethik die Rede war. Über Handlungsfreiheit könnten auch Wesen verfügen, deren `Willen` (besser: dessen innere Handlungsantriebe) vollständig kausal determiniert wären - Hobbes zufolge sind wir tatsächlich solche Wesen. Von der Handlungsfreiheit zu unterscheiden ist die Willensfreiheit, die eine Art personaler Kontrolle über die Richtung des eigenen Willens bezeichnet und deren Existenz und genaue Interpretation in der Philosophie strittig ist. 
KrV, B, S. 363

Kausalität und Willensfreiheit

GMS, S. 448
Kant nimmt an, dass Willensfreiheit von der Möglichkeit der Person abhängt, auf die Welt kausal einzuwirken, ohne dazu durch zeitlich vorausliegende `mechanische` oder innere spsychischer Kausalursachen genötigt zu sein (KpV, S. 93 ff.). Diese Art von Freiheit könne niemals Gegenstand der empirischen Erfahrung sein, weil die Annahme eines durchgängigen Kausalzusammenhangs gerade eine Bedingung der Möglichkeit von Erfahrung sei:

"Weil es ein allgemeines Gesetz [...] der Möglichkeit aller Erfahrung ist, daß alles, was geschieht, eine Ursache, mithin auch die Causalität der Ursache, die selbst geschehen oder entstanden, wiederum eine Ursache haben müsse; wodurch denn das ganze Feld der Erfahrung, so weit es sich erstrecken mag, in einem Inbegriff bloßer Natur verwandelt wird."

Gerade der Umstand, dass die Annahme einer durchgängigen kausalen Determiniertheit aller Erscheinungen eine Bedingung der Möglichkeit unserer Erfahrung ist, hat aber zugleich zur Folge, dass wir die Möglichkeit der Freiheit im Sinne der Erstursächlichkeit auch niemals aufgrund irgendwelcher Erfahrungen ausschließen können: Wenn die Tatsache, dass wir die Welt als durchgängig kausal strukturiert erfahren, aus dem Umstand resultiert, dass dies zu den Bedingungen der Möglichkeit der Erfahrung gehört, dann können wir aus ihr nicht schlussfolgern, dass die Welt an sich tatsächlich durchgängig kausal strukturiert ist. Im Übrigen stehen Freiheit und Kausalität insofern auf derselben Stufe, als auch Letztere niemals ein Gegenstand der Erfahrung sein kann. Zwar können wir Freiheit im Sinne der Erstursächlichkeit nicht erfahren. Es kann uns aber nichts davon abhalten, sie als wirklich zu denken.

Praktische Notwendigkeit der Freiheitsunterstellung: Aber warum sollten wir sie denken - warum sollten wir uns in diesem starken Sinne als frei verstehen? Eine mögliche Antwort wäre, dass wir dies in unserer Rolle als rationale Akteure unvermeidlich immer schon tun. Entsprechend führt Kant im dritten und letzten Abschnitt der Grundlegung aus, dass ein Vernunftwesen »nicht anders als unter der Idee der Freiheit handeln kann«:

"Denn in einem solchen Wesen denken wir uns eine Vernunft, die praktisch ist, d. i. Causalität in Ansehung ihrer Objecte hat. Nun kann man sich unmöglich eine Vernunft denken, die mit ihrem eigenen Bewußtsein in Ansehung ihrer Urtheile anderwärts her eine Lenkung empfinge, denn alsdann würde das Subject nicht seiner Vernunft, sondern einem Antriebe die Bestimmung der Urtheilskraft zuschreiben. Sie muß sich selbst als Urheberin ihrer Principien ansehen unabhängig von fremden Einflüssen, folglich muß sie als praktische Vernunft, oder als Wille eines vernünftigen Wesens von ihr selbst als frei angesehen werden; $d$. i. der Wille desselben kann nur unter der Idee der Freiheit ein eigener Wille sein und muß also in praktischer Absicht allen vernünftigen Wesen beigelegt werden."

Kants Argument ist also, dass wir nicht unsere Rolle deliberierender, rational zwischen verschiedenen Handlungsoptionen abwägender, Akteur/ innen ernst nehmen und dabei zugleich die Überzeugung aufrechterhal- 
ten können, dass wir in all unseren Entscheidungen durch vorausliegende Kausalursachen, etwa unsere empirischen `Neigungen`, determiniert sind (vgl. Korsgaard 1996, S. 94 ff.). Die Annahme der Willensfreiheit ist also nicht empirisch oder aufgrund theoretischer Überlegungen begründet, sondern weil sie für unsere menschliche Praxis konstitutiv ist. Wir können niemals positiv >wissen`, dass wir frei sind. So lange wir aber versuchen, uns auf vernünftige Weise in unserem Handeln zu orientieren, können wir gar nicht anders, als uns als frei zu begreifen. Dies wiederum können wir nur, indem wir uns am Prinzip der Autonomie orientieren, das zugleich das Moralprinzip ist.

Transzendentale Freiheit und Verantwortlichkeit: Ob diese Antwort Kants letztes Wort und inwieweit sie überzeugend ist, wird kontrovers diskutiert. Sie sieht sich vor allem drei Gruppen von Einwänden ausgesetzt, die hier nur knapp skizziert seien (ausführlicher z. B. Watkins 2017):

1. Eine erste Gruppe von Einwänden zielt auf den Nachweis, dass wir uns auch auf Basis einer schwächeren Freiheitskonzeption als der kantischen noch als rationale Akteur/innen verstehen könnten.

2. Eine zweite Gruppe von Einwänden legt nahe, dass Kants Freiheitsverständnis als solches problematisch ist. Tatsächlich führt Kants an den Naturwissenschaften orientierte Interpretation der Erfahrung, wonach alle empirischen Erscheinungen als kausal determiniert konzipiert werden müssen, zu der Notwendigkeit, Freiheit in eine nicht sinnlich erfahrbare snoumenale Welt - ein reines Gedankenreich - zu verlegen. Dies wirft, wie schon Fichte feststellt, die Frage auf, wie es möglich ist, innerhalb der Erscheinungswelt zurechenbare Handlungen und zurechnungsfähige Akteur/innen zu identifizieren (1962, Bd. I.3, S. 380; siehe Kap. 7.1). Empirische Kriterien dafür scheinen im Rahmen von Kants Transzendentalphilosophie nicht denkbar (eine aufschlussreiche Stellungnahme hierzu findet sich in Kants Anthropologie, AA, Bd. 7, S. $213 \mathrm{ff}$.$) .$

3. Eine dritte Gruppe von Einwänden verweist auf problematische Konsequenzen der von Kant vorgenommenen engen Kopplung zwischen Autonomie und Moralität. Zum einen ist zu klären, wie und warum freie Vernunftwesen überhaupt dem moralischen Gesetz zuwiderhandeln können. (Ein Indiz dafür, dass hier eine Schwierigkeit stecken könnte, ist ein schon in der Grundlegung feststellbares Schwanken hinsichtlich der Deutung des menschlichen Willens. Ist er »nichts anders als praktische Vernunft « oder »nicht an sich völlig der Vernunft gemäß«?, GMS, S. 412 f.). Zum anderen sollte die Erklärung jedoch so beschaffen sein, dass sie nicht die Möglichkeit ausschließt, Personen Verantwortlichkeit für Handlungen zuzuschreiben, die nicht am Moralprinzip orientiert sind - beispielsweise für selbstsüchtige Handlungen, die bewusst die Interessen anderer verletzen. Kant hat dieses Problem in seiner Schrift Die Religion innerhalb der Grenzen der blossen Vernunft (AA, Bd.6, S.1-202) durch die Annahme eines »Hangs zum Bösen« zu lösen versucht, der einerseits zur allgemeinen Menschennatur gehören, andererseits jedoch auch von jeder einzelnen Person zu verantworten sein soll, aber diese Lösung wirft eher zusätzliche Fragen auf (vgl. Bernstein 2002, S. 11-45). 
Achtung der Autonomie und Instrumentalisierungsverbot

GMS, S. 429
Ausblick: Ungeachtet der angedeuteten Schwierigkeiten ist Kants Beitrag zum moralphilosophischen Denken nicht zu überschätzen. Kant bemüht sich um eine Interpretation und Begründung der Moral, die dem von Hume hervorgehobenen Problem des Übergangs vom Sein zum Sollen konsequenter Rechnung trägt als Humes eigene Tugendlehre. Vor diesem Hintergrund hat er zentrale Probleme benannt, denen sich eudaimonistische, instrumentalistische oder sentimentalistische Ansätze gegenübergestellt sehen. Kants Interpretation moralischer Pflichten als >kategorisch vorrangig gegenüber allen anderen praktischen Erwägungen prägt die Diskussion weit über den eigenen Schulzusammenhang hinaus (Birnbacher 2013, Kap. 1).

Kants Bemühen, Prinzipien der praktischen Vernunft nicht in der kontemplativen Einstellung eines Naturforschers oder Moralpsychologen zu gewinnen, sondern durch die Reflexion auf die Bedingungen unseres Deliberierens und Handelns selbst, etabliert ein neues Modell ethischen Denkens, das seither in vielfältigen Varianten weiterentwickelt wird. Es führt ihn auch zu einem modernen Moralverständnis, das personale Verantwortung und Autonomie und damit die Würde des Individuums zentral stellt. Aus Kants Autonomie- und Moralprinzip folgt nämlich, dass wir die eigene Autonomie nur realisieren können, indem wir zugleich die Autonomie aller anderen wahren. Denn um unsere Autonomie zu realisieren (statt tatsächlich zum Sklaven unserer Neigungen zu werden) müssen wir nach Maximen handeln, die wir als allgemeine Gesetze wollen können. Wie gezeigt kann dies jedoch nur bedeuten, nach Maximen zu handeln, die alle Betroffenen vernünftigerweise als allgemeine Gesetze akzeptieren können. Das impliziert eine Pflicht zur moralischen Anerkennung von Personen. Sie wird auch in der sogenannten Selbstzweckformel des Kategorischen Imperativs zum Ausdruck gebracht, die eine Instrumentalisierung anderer Menschen verbietet:

"Handele so, daß du die Menschheit sowohl in deiner Person, als in der Person eines jeden anderen jederzeit zugleich als Zweck, niemals bloß als Mittel brauchst."

Diese Forderung drückt eine Intuition des unbedingten Respekts vor der Urteilsautonomie aller Personen aus, von der unser heutiges Moralverständnis ebenso wesentlich geprägt ist wie unsere auf dem Prinzip der Menschenwürde basierende Verfassungsordnung.

\section{Siglenverzeichnis}

AA - Akademieausgabe (Kant 1902 ff.)

DK - Diels/Kranz, Die Fragmente der Vorsokratiker

GMS - Grundlegung zur Metaphysik der Sitten (Kant, AA, IV, S. 385-463)

$\mathrm{KpV}$ - Kritik der praktischen Vernunft (Kant, AA, V, S. 1-163)

$\mathrm{KrV}$ - Kritik der reinen Vernunft (Kant, 2. Aufl. (B) AA, III, 1. Aufl. (A) AA IV, $1-252)$

KdU - Kritik der Urteilskraft (Kant, AA, V, S. 165-485)

MdS - Metaphysik der Sitten (Kant, AA, VI, S. 203-493)

SEP - Stanford Encyclopedia of Philosophy (Zalta) 


\section{Einführende Literatur}

Guyer, Paul (Hrsg.): The Cambridge Companion to Kant and Modern Philosophy. Cambridge 2006.

Höffe, Otfried: Immanuel Kant. München 1992.

Irrlitz, Gerd (Hrsg.): Kant-Handbuch: Leben und Werk. Stuttgart 2002.

Kant, Immanuel: Grundlegung zur Metaphysik der Sitten, mit einem Kommentar von Christoph Horn, Corinna Mieth und Nico Scarano. Frankfurt a. M. ${ }^{2} 2010$.

Reath, Andrews/Timmermann, Jens (Hrsg.): Kant's »Critique of Practical Reason«: A Critical Guide. Cambridge 2013.

Schönecker, Dieter/Wood, Allen W.: Kants »Grundlegung zur Metaphysik der Sitten«: Ein einführender Kommentar. Paderborn ${ }^{4} 2011$.

Timmermann, Jens: Kant's Groundwork of the Metaphysics of Morals: A Commentary. Cambridge 2007.

Tugendhat, Ernst: Vorlesungen über Ethik. Frankfurt a. M. 1993.

\section{Zitierte und weiterführende Literatur}

Allison, Henry E.: »Morality and Freedom: Kant’s Reciprocity Thesis«. In: The Philosophical Review 95/3 (1986), S. 393-425.

Bernstein, Richard J.: Radical Evil: A Philosophical Interrogation. Cambridge 2002.

Broad, C. D.: Five Types of Ethical Theory [1930]. London 2013.

Fichte, Johann G.: Gesamtausgabe der Bayerischen Akademie der Wissenschaften. Stuttgart-Bad Cannstatt $1962 \mathrm{ff}$.

Habermas, Jürgen: Die Einbeziehung des Anderen. Frankfurt a. M. 1996.

Hegel, Georg W.: Werke in zwanzig Bänden. Frankfurt a. M. 1970.

Höffe, Otfried: »Kants kategorischer Imperativ als Kriterium des Sittlichen«. In: Zeitschrift für philosophische Forschung 31/3 (1977), S. 354-384.

Höffe, Otfried: Kategorische Rechtsprinzipien: Ein Kontrapunkt der Moderne. Frankfurt a. M. 1990.

Höffe, Otfried: »Kants nichtempirische Verallgemeinerung: Zum Rechtsbeispiel des falschen Versprechens«. In: Ders. (Hrsg.): Grundlegung zur Metaphysik der Sitten: Ein kooperativer Kommentar. Frankfurt a. M. ${ }^{3} 2000$, S. 206-233.

Hume, David: A Treatise of Human Nature. Hrsg. von David Fate Norton und Mary J. Norton. Clarendon Hume Edition Series, Bd. 1: Texts. Oxford/New York 2007.

Kant, Immanuel: Gesammelte Schriften. Hrsg. von der Königlich Preußischen Akademie der Wissenschaften. Berlin $1902 \mathrm{ff}$.

Kersting, Wolfgang: Wohlgeordnete Freiheit: Immanuel Kants Rechts- und Staatsphilosophie. Frankfurt a. M. 1993.

Kohlberg, Lawrence: Essays in Moral Development. Cambridge 1981.

Korsgaard, Christine M.: The Sources of Normativity. Cambridge 1996.

Neusner, Jacob/Chilton, Bruce B.: The Golden Rule: The Ethics of Reciprocity in World Religions. London/New York 2009.

Parfit, Derek: On What Matters. 2 Bände. Oxford/New York 2011.

Potter, Nelson T./Timmons, Mark: Morality and Universality: Essays on Universalizibility. Dordrecht u. a. 1985.

Ross, David: Kant's Ethical Theory: A Commentary on the Grundlegung zur Metaphysik der Sitten. Oxford 1954.

Rousseau, Jean-Jacques: Collection complète des oeuvres de J. J. Rousseau. Genève 1762.

Singer, Marcus G.: Generalization in Ethics: An Essay in the Logic of Ethics, with the Rudiments of a System of Moral Philosophy. London 1963.

Singer, Marcus G.: Golden Rule. In: Becker, Lawrence C./Becker, Charlotte B. (Hrsg.): Encyclopedia of Ethics. New York/London ${ }^{2} 2001$, Bd. 1, S. 61.

Smart, J. J. C.: »An Outline of a System of Utilitarian Ethics«. In: Smart, J. J.C./ Williams, Bernard: Utilitarianism: For and Against. Cambridge 1973, S. 3-74. 
Watkins, Eric (Hrsg.): Kant on Persons and Agency. Cambridge 2017.

Wattles, Jeffrey: The Golden Rule. Oxford/New York 1997.

Werner, Micha H.: Diskursethik als Maximenethik: Von der Prinzipienbegründung zur Handlungsorientierung. Würzburg 2003.

Wimmer, Reiner: Universalisierung in der Ethik: Analyse, Kritik und Rekonstruktion ethischer Rationalitätsansprüche. Frankfurt a. M. 1980.

Wood, Allen W.: Kant's Ethical Thought. Cambridge 1999.

Zalta, Edward N. (Hrsg.): The Stanford Encyclopedia of Philosophy. In: https:// plato.stanford.edu/.

Open Access Dieses Kapitel wird unter der Creative Commons Namensnennung 4.0 International Lizenz (http://creativecommons.org/licenses/by/4.0/deed.de) veröffentlicht, welche die Nutzung, Vervielfältigung, Bearbeitung, Verbreitung und Wiedergabe in jeglichem Medium und Format erlaubt, sofern Sie den/die ursprünglichen Autor(en) und die Quelle ordnungsgemäß nennen, einen Link zur Creative Commons Lizenz beifügen und angeben, ob Änderungen vorgenommen wurden.

Die in diesem Kapitel enthaltenen Bilder und sonstiges Drittmaterial unterliegen ebenfalls der genannten Creative Commons Lizenz, sofern sich aus der Abbildungslegende nichts anderes ergibt. Sofern das betreffende Material nicht unter der genannten Creative Commons Lizenz steht und die betreffende Handlung nicht nach gesetzlichen Vorschriften erlaubt ist, ist für die oben aufgeführten Weiterverwendungen des Materials die Einwilligung des jeweiligen Rechteinhabers einzuholen. 